\title{
Hanani-Tutte and Related Results
}

\author{
Marcus Schaefer \\ Department of Computer Science \\ DePaul University \\ Chicago, Illinois 60604, USA \\ mschaefer@cdm.depaul.edu
}

August 16, 2011

We are taking the view that crossings of adjacent edges are trivial, and easily got rid of.

Bill Tutte

We interpret this sentence as a philosophical view and not a mathematical claim.

László Székely

\begin{abstract}
We investigate under what conditions crossings of adjacent edges and pairs of edges crossing an even number of times are unnecessary when drawing graphs. This leads us to explore the Hanani-Tutte theorem and its close relatives, emphasizing the intuitive geometric content of these results.
\end{abstract}

\section{The Hanani-Tutte Theorem in The Plane}

In 1934, Hanani [15] published a paper which-in passing - established the following result:

Any drawing of a $K_{5}$ or a $K_{3,3}$ contains two independent edges crossing each other oddly. ${ }^{1}$

Since by Kuratowski's theorem every non-planar graph contains a subdivision of $K_{5}$ or $K_{3,3}$, Hanani's observation implies that any drawing of a nonplanar graph contains two vertex-disjoint paths that cross an odd number of times and therefore two independent edges that cross oddly - one in each path. This consequence was first explicitly stated by Tutte [49]. ${ }^{2}$

\footnotetext{
${ }^{1}$ The result can be hard to find even if one reads German. It is stated as (1) on page 137 of the article and mainly an application of methods developed by Flores [20,62. Kolloqium].

${ }^{2}$ The theorem is generally known as the Hanani-Tutte theorem, though Levow [17] calls it the "van Kampen-Shapiro-Wu characterization of planar graphs" emphasizing the parallel history of the theorem in algebraic topology (ignoring Flores, however). In a recent paper [34] we introduced the name "strong Hanani-Tutte theorem" to distinguish it from a weaker version that is also often called the Hanani-Tutte theorem in the literature.
} 
Theorem 1.1 (The (Strong) Hanani-Tutte Theorem). Any drawing of a nonplanar graph contains two independent edges that cross oddly.

Equivalently, but more spectacularly, the theorem can be phrased as saying that if we can draw a graph so that every two independent edges cross evenly, then the graph is planar (we study the algorithmic content of this statement in Section 1.4). Since the reverse direction is immediate, the Hanani-Tutte theorem can be viewed as a characterization of planarity.

In this paper we bring together different versions and applications of the Hanani-Tutte theorem to show that the result of Hanani-Tutte deserves the epithets "remarkable" and "beautiful" [27, 29]. We acknowledge the roots of Hanani-Tutte in the literature of algebraic topology, but this paper will take an intuitive, geometric approach which proves sufficient as long as we restrict ourselves to two-dimensional surfaces.

Some conventions used in this paper: When we speak of drawings of graphs we do not distinguish between an abstract edge and the arc representing it in the plane; or a vertex and the point it is located at. We will simply use "edge" and "vertex" for both concepts; we use topological graph when we want to emphasize that we are considering an abstract graph together with a drawing. We require drawings of graphs to fulfill the standard properties: there are only finitely many intersections, a vertex does not lie in the interior of an edge, no two vertices lie in the same location, and at most two edges intersect at any interior intersection point. Interior intersection points of edges come in two flavors: crossings, if the edges cross at that point, or touching points if the edges touch. A common endpoint of two edges is considered an intersection point, but it is neither a crossing nor a touching point.

\subsection{The Weak Hanani-Tutte Theorem, or, Even Crossings Don't Matter}

The Hanani-Tutte theorem is often stated and used in a weak form. Call an edge in a drawing even if it crosses every other edge an even number of times (including 0 times). If a graph can be drawn so that all its edges are even, then the graph is planar. This weak version of the Hanani-Tutte theorem is easier to prove than the strong version, and yields a stronger conclusion: the graph can be embedded in the plane without changing its rotation system. The rotation at a vertex is the cyclic ordering of ends of edges at that vertex, the rotation system is the collection of rotations of all vertices.

Theorem 1.2 (Weak Hanani-Tutte). If a graph can be drawn so that all its edges are even, then the graph is planar and can be embedded without changing the rotation system.

The assumption of the weak Hanani-Tutte theorem can be weakened: it is enough to require that in the drawing every even subgraph of $G$, that is, a 
subgraph all of whose vertices have even degree, has an even number of selfcrossings. This form of the theorem was suggested and proved by Loebl and Masbaum in their study of Norine's conjecture [18].

Theorem 1.3 (Loebl, Masbaum). If a graph can be drawn so that every even subgraph has an even number of self-crossings, then the graph can be embedded in the plane without changing the rotation system.

Or, as Loebl and Masbaum phrase it: "Even drawings don't help". Theorem 1.3 immediately implies Theorem 1.2. We include an easy proof of Theorem 1.3 using geometric rather than homological methods.

Proof. Suppose we are given a drawing of the graph so that $(*)$ every even subgraph has an even number of self-crossings.

We can assume that the graph is connected: Adding an edge between two components of the graph does not affect $(*)$ since that edge cannot be part of any even subgraph (it would, in each of the components, be incident to a subgraph of odd total degree, which contradicts the handshake lemma). Repeating this, we obtain a connected graph fulfilling $(*)$. If the new graph is embeddable without changing its rotation system, then the original graph can be embedded with its original rotation system (delete the additional edges). We prove the result for connected graphs by induction on the number of vertices and edges. To make the induction work, we allow multiple edges and loops.

If there is a non-loop edge $e=u v$ contract it by moving $v$ along $e$ towards $u$, eventually identifying $u$ and $v$ and merging the rotations of $u$ and $v$ as shown in Figure 1. We presently argue that $(*)$ remains true, so by the inductive assumption, the new graph can be embedded without changing its rotation; but then we can split $u=v$ into two vertices again and move them apart slightly, recovering the original rotations of $u$ and $v$ and reinserting the edge $e=u v$ without introducing any crossings.
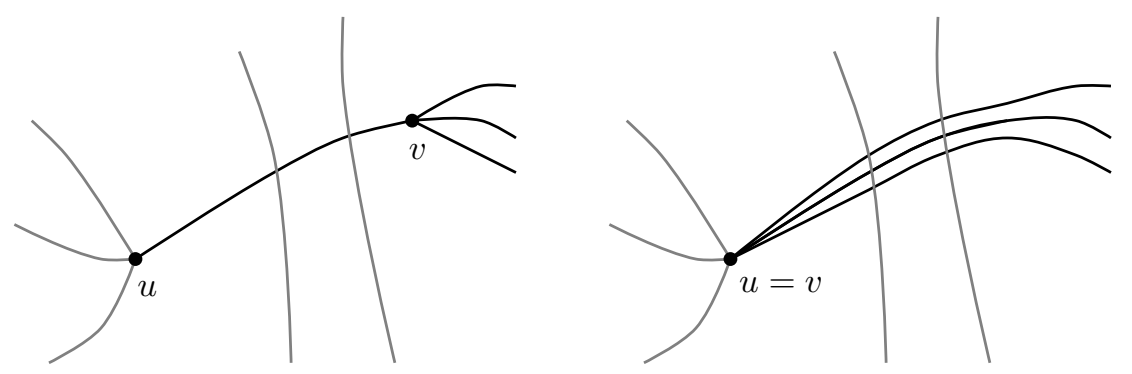

Figure 1: Geometrically contracting edge $e=u v$ towards $u$.

The contraction of $e$ does not affect $(*)$ : let $H$ be an arbitrary even subgraph before the contraction. If $e \notin E(H)$, then, since the degree of $H$ at $v$ is even, an even number of edges of $H$ is pulled along $e$, so any crossing of $e$ leads to an even number of crossing with $H$, so the number of self-crossings of $H$ remains 
even. The same conclusion holds if $e \in E(H)$. In this case we are pulling an odd number of edges of $H$ along $e$, but $e$ itself belongs to $H$, so if $v$ is pulled through a crossing with some edge $f \in E(H)$, the odd number of crossings added is balanced by the single crossing between $e$ and $f$ that is removed, so that the overall parity remains even.

Since we started with a connected graph, we are left with the case of a single vertex with loops. Any loop by itself is an even subgraph, so it has an even number of self-crossings. Since any two loops also form an even subgraph, any two loops must cross each other an even number of times. Pick any loop $e$ whose ends are closest in the rotation; the ends of $e$ must be consecutive (any loop starting between the two ends of $e$ would also have to end between the two ends, but then we would have chosen it over $e$ ). Remove $e$ and draw the remaining graph by induction (with the same rotation). We can then reinsert $e$ at its original place in the rotation without introducing any crossings. This completes the proof.

The Loebl-Masbaum result no longer holds if we only require that every cycle has an even number of self-crossings: take two cycles sharing a single vertex so that the ends of the cycle alternate at the vertex. While this graph is planar it cannot be drawn without changing its rotation system. A simple modification of the theorem is true, however.

Theorem 1.4. If a graph can be drawn so that every cycle has an even number of self-crossings, then the graph can be embedded in the plane without changing the rotation system of any 2-connected block of the graph. (Only the rotations at cut-vertices need to be adjusted: make the ends of edges belonging to the same 2-connected block consecutive in the rotation without otherwise changing the ordering of ends belonging to the same block.)

Even though Theorem 1.4 leads to a change in rotation, it has the flavor of the weak Hanani-Tutte theorem; we can ask whether it is sufficient to assume that every cycle has an even number of independent crossings to guarantee planarity. Theorem 1.16 in the next section answers that question.

In the proof of the theorem, we will contract edges only partially, namely, up to a point where they are free of crossings. ${ }^{3}$

Proof of Theorem 1.4. We show that any 2-connected graph fulfilling the conditions of the theorem can be embedded without changing its rotation system. The general result then follows.

First note that contracting an edge, even partially, does not affect the parity of the number of crossings along any cycle, since a cycle always has even degree at every vertex. So we can pick a spanning tree $T$ of the graph and partially contract edges in a breadth-first (or depth-first) order towards the root of the tree so that all edges of $T$ are entirely free of crossings. This might introduce self-crossings along edges in $E(G)-E(T)$, but since each such edge forms a

\footnotetext{
${ }^{3}$ Partial contractions were used by Černý [9] in his proof of the weak Hanani-Tutte theorem in the plane.
} 
cycle with a path in $T$, it must have an even number of self-crossings, so we can remove all self-crossings of edges without changing the parity of crossings along any cycle. At this point the only crossings are between distinct edges in $E(G)-E(T)$. We claim that any two such edges $e, f \in E(G)-E(T)$ cross evenly: for a contradiction assume that $e$ and $f$ cross oddly. Let $P_{e}$ and $P_{f}$ be the sub-paths of $T$ connecting the endpoints of $e$ and $f$ and $C_{e}=P_{e} \cup\{e\}$, $C_{f}=P_{f} \cup\{f\}$. First consider the case that $e$ and $f$ are independent. Since $e$ and $f$ cross oddly, but two closed curves in the plane always cross evenly, $C_{e}$ and $C_{f}$ must have at least a vertex in common. If $C_{e}$ and $C_{f}$ share an edge, we argue as follows: the symmetric difference $C^{\prime}=C_{e} \triangle C_{f}$ is a cycle consisting of $e, f$ and crossing-free edges from $T$. Since by assumption $C^{\prime}$ has an even number of self-crossings, $e$ and $f$ must cross evenly. If $C_{e}$ and $C_{f}$ share only a vertex, say $v$, then $C_{e}-v$ and $C_{f}-v$ are disjoint, so since $G$ is 2-connected, there must be an edge $g \notin E(T)$ connecting them. Consider the following cycles pictured in Figure 2:

$C_{e, g}$ : start at the endpoint of $g$ lying on $C_{e}$, follow $C_{e}$ to $e$, traverse $e$, follow $C_{e}$ to $v$, follow $C_{f}$ to the other endpoint of $g$, traverse $g$,

$C_{f, g}$ : start at the endpoint of $g$ lying on $C_{f}$, follow $C_{f}$ to $f$, traverse $f$, follow $C_{f}$ to $v$, follow $C_{e}$ to the other endpoint of $g$, traverse $g$,

$C^{\prime}$ : start at the endpoints of $g$ lying on $C_{e}$ and $C_{f}$, follow $C_{e}$ to $v$ traversing $e$ and follow $C_{f}$ to $v$ traversing $f$; add $g$.
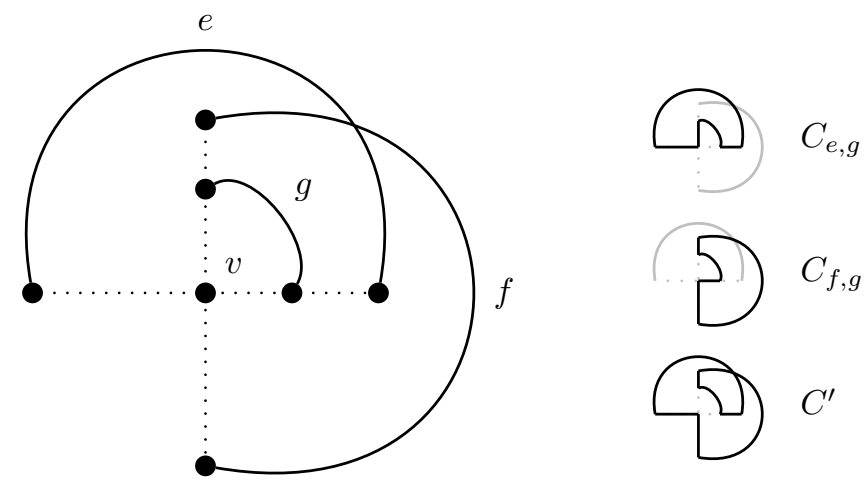

Figure 2: Cycles $C_{e, g}, C_{f, g}$ and $C^{\prime}$ in case $e$ and $f$ are independent.

Now $C_{e, g}$ and $C_{f, g}$ are even, so both $e$ and $g$ as well as $f$ and $g$ cross evenly (all other edges in these cycles are crossing-free). Since $C^{\prime}$ is even, this means that $e$ and $f$ also have to cross evenly.

In case $e$ and $f$ share an endpoint $v$, let $T_{e}$ and $T_{f}$ be the components of $T-\{v\}$ containing the other endpoints of $e$ and $f$. If $T_{e}=T_{f}$, then $T-\{v\}$ contains a path $P$ connecting the two endpoints of $e$ and $f$ which are different 
from $v$. Now the cycle $P \cup\{e\} \cup\{f\}$ is even; since all edges of $P$ are free of crossings, this implies that $e$ and $f$ cross evenly.

So we can assume that $T_{e} \neq T_{f}$. Since $G$ is 2 -connected, there must be an edge $g$ between $T_{e}$ and $T_{f}$. With $g$ we can construct three cycles as shown in Figure 3:

$C_{e, g}$ : start at $v$, follow $e$, follow $T_{e}$ to $g$, follow $g$, follow $T_{f}$ to $v$,

$C_{f, g}$ : start at $v$, follow $f$, follow $T_{f}$ to $g$, follow $g$, follow $T_{e}$ to $v$,

$C^{\prime}$ : start at $v$, follow $e$, follow $T_{e}$ to $g$, follow $g$, follow $T_{f}$ to $f$, follow $f$ back

to $v$.
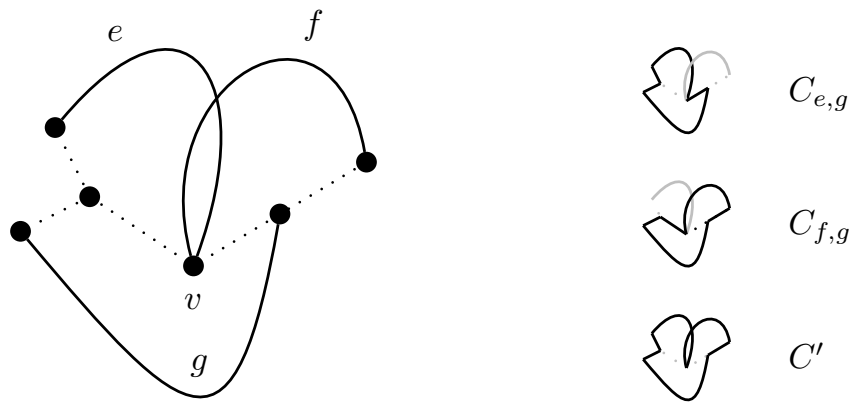

Figure 3: Cycles $C_{e, g}, C_{f, g}$ and $C^{\prime}$ in case $e$ and $f$ are adjacent.

Since $C_{e, g}$ is even, $e$ and $g$ cross evenly (by an argument similar to the one above); by the same token, the evenness of $C_{f, g}$ implies that $f$ and $g$ cross evenly. Finally, since $C^{\prime}$ is even, and, we assumed $e$ and $f$ cross oddly, $e$ or $f$ must cross oddly with $g$, but we saw that this is not the case, so $e$ and $f$ cross evenly.

In other words, all edges in the current drawing of $G$ are even. By the weak Hanani-Tutte theorem, we can then embed $G$ in the plane without changing its rotation system.

Theorem 1.4 suggests a more general family of Hanani-Tutte type results: if we know that all subgraphs belonging to some family of graphs have an even number of self-crossings, what does this tell us about the graph? To guarantee planarity we saw that it is enough to look at pairs of edges (weak Hanani-Tutte), pairs of independent edges (strong Hanani-Tutte), even subgraphs (Loebl-Masbaum), cycles (Theorem 1.4). What other families of graphs guarantee planarity?

Paths furnish a trivial example: if all paths have an even number of self-crossings, then all edges are even: consider two edges $e$ and $f$, and let $P$ be a shortest path containing both $e$ and $f$ (so they must be the first and last edge of the path). Since $P-\{e, f\}, P-\{e\}, P-\{f\}$ as well as $P$ are even, $e$ and $f$ have to cross evenly. Hence all edges are even, and the graph is planar. 
Stars on the other hand, do not appear very promising at first: any drawing of a graph which minimizes the number of crossings has no crossings between adjacent edges, so for any graph all stars can be made free of self-crossings. Looking at pairs of stars trivializes the problem: any two edges are a pair of stars, so by the weak Hanani-Tutte theorem, the graph is planar. However, there is an interesting variant hiding here: what happens if we consider pairs of maximal stars?

Question 1.5. If $G$ is drawn so that every union of two maximal stars in $G$ has an even number of self-crossings, is $G$ planar?

Note that this fails for the torus: take a toroidal grid $C_{n} \square C_{n}$ and add a pair of diagonal edges to each square. Then the union of two maximal stars will always contain 0 or 2 self-crossings.

Other families of graphs worth exploring might be triples of (independent) edges and $\Theta$-graphs, that is, graphs consisting of three internally disjoint paths connecting the same pair of vertices.

Even if we do not get planarity, we can still ask whether requiring certain subgraphs in the drawing to have an even number of self-crossings allows us to draw any conclusions about the graph. One might, for example, ask extremal questions. We are not aware of any extremal results of this particular form, however, there are several very similar extremal results we will discuss in Section 3.2. There is a result by Pach and Tóth [31] worth mentioning in this context: a topological graph for which any set of $k \geq 2$ independent edges contains two edges that cross evenly has at most $O\left(n \log ^{4 k-8} n\right)$ edges. For $k=2$ this is a consequence of the strong Hanani-Tutte theorem. ${ }^{4}$

On the structural side there is Norine's fine characterization of Pfaffian graphs [22]: he shows that a graph is Pfaffian if and only if it has a drawing in which every perfect matching has an even number of self-crossings (edges are not allowed to self-intersect in this characterization).

One might ask which natural properties of graphs are invariant under weakening crossing-free to even or independently even, where an edge is independently even if it crosses every non-adjacent edge an even number of times. Suppose, for example, that we have a drawing of a graph in which all vertices lie on the boundary of the same region, and all edges are independently even. Then the graph is outerplanar. So outerplanarity survives the weakening of crossing-free to independently even. (The proof is simple: to the region containing all vertices on the boundary, add a new vertex and connect it to all other vertices by crossing-free edges. All edges in the resulting drawing are independently even, so by the strong Hanani-Tutte theorem the graph is planar. Removing the new vertex from the planar drawing yields an outerplanar drawing of the graph.) On the other hand, the notion of crossing number is not invariant under replacing crossing-free with even or independently even as we will see in Section 3.4.

Another example is furnished by a result of Pach and Tóth's on $x$-monotone drawings; call a drawing $x$-monotone if all its edges are $x$-monotone, that is, functions on an interval. It is known that every $x$-monotone embedding of a graph can be turned into a straight-line embedding without changing the $x$ coordinates of any vertex $[11,30]$.

\footnotetext{
${ }^{4}$ This result, with a slightly weaker bound, was rediscovered and used in [6].
} 
Theorem 1.6 (Pach, Tóth [30]). If all edges in an x-monotone drawing of a graph are even, then the graph has a straight-line embedding in which every vertex keeps its $x$-coordinate.

Does the result remain true if we only require edges to be independently even rather than even? Since $x$-monotone embeddings can be turned into straight-line embeddings without changing $x$-coordinates, it would be sufficient to establish the following conjecture.

Conjecture 1.7. If all edges in an x-monotone drawing of a graph are independently even, then there is an x-monotone embedding of the graph in which every vertex keeps its $x$-coordinate.

The proof of Theorem 1.6 uses the Cairns-Nikolayevsky proof of Theorem 1.2; it is not immediately clear whether the redrawing techniques we have used in this section can be adapted to establish Theorem 1.6 or the conjecture.

The weak version of Hanani-Tutte is a simple but popular form of the theorem. As such it has been discovered independently a couple of times. Cairns and Nikolayevsky used homology theory and intersection forms to prove the result for arbitrary surfaces [7]; an intuitive geometric proof, again for arbitrary surfaces, can be found in [36]. The proof for the plane was independently found by Cerný [9]. It also follows from a redrawing result of Pach and Tóth [29] which we discuss in Section 3.4.

\subsection{Planarity Criteria and Weak Hanani-Tutte}

In a way all versions of Hanani-Tutte are planarity criteria, but the two variants we explore in this section are special in that they depend on the rotation system of the graph only, and not on the particular drawing. The first criterion is due to Cairns and Nikolayevsky [7] and is implicit in their proof of the weak HananiTutte theorem. To state the result we need to define a new notion of the number of crossings between two cycles of a graph: Consider two cycles $C_{1}$ and $C_{2}$ and let $P$ be a maximal path in $C_{1} \cap C_{2}$; contract $P$ to a single vertex. If the ends of $C_{1}$ and $C_{2}$ alternate at that vertex, we say the cycles cross in $P$ and count this as one crossing; otherwise $C_{1}$ and $C_{2}$ touch in $P$ and we do not count this as a crossing. Let $\sigma\left(C_{1}, C_{2}\right)$ be the total number of crossings - in this sensebetween $C_{1}$ and $C_{2}$. Note that we do not count crossings between edges from $C_{1}$ and $C_{2}$, so $\sigma\left(C_{1}, C_{2}\right)$ is completely determined by a rotation system of the graph.

Theorem 1.8 (Cairns, Nikolayevsky [7]). If a graph can be drawn so that $\sigma\left(C_{1}, C_{2}\right)$ is even for every two cycles $C_{1}, C_{2}$ in the graph, then the graph can be embedded in the plane without changing the rotation system.

Theorem 1.8 is easily seen to imply the weak Hanani-Tutte theorem. A proof of the theorem can proceed along the same lines as the proof of Theorem 1.3: The parity of $\sigma\left(C_{1}, C_{2}\right)$ is not affected by contractions of edges, so we can 
contract the graph to a single vertex with loops; now by assumption any two loops cross an even number of times (in the traditional sense of crossings); but then we can embed the graph as we did in the proof of Theorem 1.3.

The second planarity criterion is due to Lovász, Pach and Szegedy [19] and, like the Cairns-Nikolayevsky criterion, arose in the study of thrackles (of which more in Section 3.3). Recall that a $\Theta$-graph is a pair of vertices connected by three internally disjoint paths. In a drawing of a $\Theta$-graph the cyclic clockwise ordering in which the three paths end at the two vertices is either the same or reversed; if the order is reversed, we call the $\Theta$-graph a converter. A plane $\Theta$-graph is always a converter.

Theorem 1.9 (Lovász, Pach, and Szegedy [19]). A graph is planar if and only if it can be drawn so that every $\Theta$-subgraph is a converter. In that case, the graph can be embedded in the plane without changing the rotation system of any 2-connected block of the graph (only the rotations at cut-vertices need to be changed).

Rather than establishing the planarity criterion from scratch (which isn't very hard assuming Kuratowski's theorem), we will show that it is really an incarnation of Theorem 1.2. Indeed, this is how we obtain the conclusion about the rotation system, which is not part of the original result of Lovász, Pach and Szegedy (using Kuratowski's theorem doesn't allow any conclusions about the rotation). In the proof we use a characterization of $\Theta$-graphs in terms of self-crossings.

Lemma 1.10. A $\Theta$-graph is a converter if and only if it has an even number of cycles with an odd number of self-crossings.

The lemma could be established using exhaustive case-analysis; instead we opt to obtain it as a consequence of Theorem 1.4.

Proof. If a $\Theta$-graph is drawn so that it has an even number of cycles with an odd number of self-crossings, there are either no or two cycles with an odd number of self-crossings. In case there are two cycles with an odd number of crossings, the two cycles share one of the three paths; introduce a self-crossing (within one edge) along that path. Hence, we can assume that all cycles have an even number of self-crossings. By Theorem 1.4, the graph can be embedded in the plane with the same rotation system, so it must be a converter.

To establish the other direction, swap the ends of two edges at one of the two vertices defining the $\Theta$-graph; this changes the parity of the number of cycles with an odd number of self-crossings, so it becomes even. Then by the argument we made in the first paragraph, the modified graph is a converter, which means that the original graph (before swapping the ends) is not.

$\Theta$-graphs are not closed under contracting edges, leading to problems with proofs centered around contraction. To address this issue, we introduce $\vartheta$-graphs as graphs resulting from completely contracting one of the paths of a $\Theta$-graph; 
in other words, a $\vartheta$-graph is a vertex with two closed, internally disjoint paths starting and ending at that vertex. We call a $\vartheta$-graph a converter if the ends of the two closed paths do not alternate at the shared vertex. (If we contract one of the paths of a $\Theta$-graph which is a converter, the resulting $\vartheta$-graph is a converter.) It is easy to see that a $\vartheta$-graph is a converter if and only if its two closed paths cross an even number of times (not counting the shared vertex $v$ ).

Lemma 1.11. If every $\Theta$-subgraph in a drawing of a 2 -connected graph (without loops) is a converter, then every $\vartheta$-subgraph in the drawing is a converter.

Proof. Fix a drawing of the graph and assume that every $\Theta$-subgraph is a converter. Pick a $\vartheta$-subgraph at some vertex $v$ formed by two closed paths $P$ and $Q$. Since the graph is 2-connected, there must be a path connecting $P-v$ and $Q-v$ (each of these paths contains at least one vertex, since the graph contains no loops). Let $R$ be a shortest such path with endpoints $p \in P$ and $q \in Q$. Now $P$ consists of two (proper) $v, p$-paths $P_{1}, P_{2}$ and $Q$ of two $v, q$-paths $Q_{1}, Q_{2}$. With these pieces we can build two $\Theta$-graphs between $v$ and $p: P_{1}, P_{2}, R+Q_{1}$ and $P_{1}, P_{2}, R+Q_{2}$. By assumption, both of the $\Theta$-graphs are converters, so the cyclic clockwise orderings of the three paths at $v$ and $p$ must be reversed. Let us assume that paths $P_{1}, R, P_{2}$ occur in this clockwise, cyclic ordering at $p$ (the other case $P_{2}, R, P_{1}$ is analogous). But then the two continuations of $R$ ( $Q_{1}$ and $Q_{2}$ ) must each occur between $P_{2}$ and $P_{1}$ in the clockwise cyclic ordering at $v$ since the two $\Theta$-graphs are converters. Hence, $Q_{1}$ and $Q_{2}$ are consecutive at $v$, which implies that the $\vartheta$-subgraph formed by $P$ and $Q$ is a converter.

With these results we are in a position to show that Theorem 1.9 is a variant of the weak Hanani-Tutte theorem.

Proof of Equivalence of Theorem 1.9 and Theorem 1.2. In a drawing in which every two edges cross an even number of times, every $\Theta$-subgraph is a converter by Lemma 1.10 (every cycle has an even number of self-crossings and we can assume that edges are free of self-crossings). This shows that Theorem 1.9 implies Theorem 1.2.

To see the other direction, let the graph be drawn so that every $\Theta$-subgraph is a converter. It is sufficient to prove the result for 2-connected graphs, and then recombine the drawings at the cut-vertices. As we did in the proof of Theorem 1.4, we can partially contract the edges of a spanning tree $T$ so that all edges of $T$ are free of crossings. Let $e$ and $f$ be any two edges of the graph. We show that $e$ and $f$ cross an even number of times. This is obvious if either one of them belongs to $E(T)$, so we can assume that $e, f \notin E(T)$ and there are cycles $C_{e} \subseteq T+e$ and $C_{f} \subseteq T+f$. If $C_{e}$ and $C_{f}$ do not share a vertex, they must cross an even number of times in the plane, so $e$ and $f$ cross evenly; if $C_{e}$ and $C_{f}$ share a vertex, but not an edge, then $C_{e} \cup C_{f}$ is a $\vartheta$-graph, so by Lemma 1.11 it is a converter, and $e$ and $f$ cross evenly. Finally, if $C_{e}$ and $C_{f}$ share more than one vertex, they share a non-empty path, and $C_{e} \cup C_{f}$ is a $\Theta$-graph which, by assumption, is a converter. But then $e$ and $f$ cross evenly by Lemma 1.10 . 


\subsection{Strong Hanani-Tutte and Cycles}

Maybe the shortest and most elegant proof of the Hanani-Tutte theorem is due to Kleitman [16]. He essentially establishes Hanani's original result using an intuitive geometric approach by showing that all drawings of $K_{2 i+1}$ and $K_{2 i+1,2 j+1}$ have an odd number of independent crossings. ${ }^{5}$ Together with Kuratowski's theorem this yields the strong Hanani-Tutte theorem in the same way that Hanani's result did.

Archdeacon and Richter later showed that $K_{2 i+1}$ and $K_{2 i+1,2 j+1}$ are the only graphs for which the parity of independent crossings is the same in all drawings [2].

We do not include Kleitman's proof since we will show how to obtain an even stronger version of the Hanani-Tutte theorem using ideas similar to his. The following lemma has been used and stated in many forms, but its core ideas really go all the way back to van Kampen [54]. An $(e, v)$-move consists of deforming a small part of $e$, moving it close to $v$ and then pulling it over $v$. It changes the parity of crossing of $e$ with every edge incident to $v$, but with no other edge, see Figure 4. A rotation swap consists of swapping the order of two consecutive ends at a vertex.
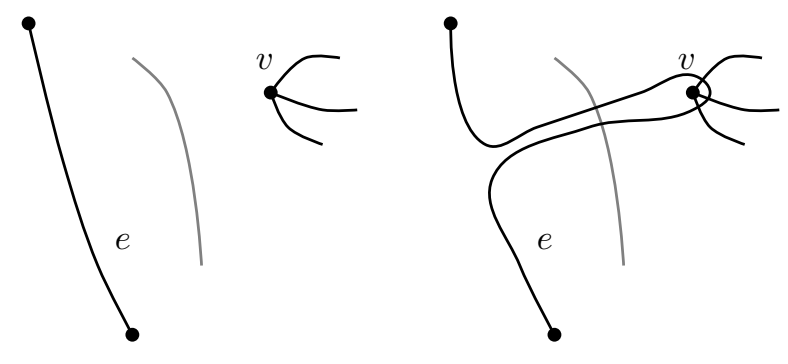

Figure 4: Performing an $(e, v)$-move.

Lemma 1.12. Given two drawings $D_{1}$ and $D_{2}$ of the same graph, there is a set of $(e, v)$-moves and rotation swaps that can be applied to $D_{1}$ so that the resulting drawing $D_{1}^{\prime}$ has the same parity of crossing between every pair of edges as $D_{2}$.

Proof. We follow Kleitman's argument [16]: start with $D_{1}$. By deforming the plane, we can assume that each vertex has the same location in $D_{1}$ and $D_{2}$. Now continuously deform each edge $e$ from its drawing in $D_{1}$ to its drawing in $D_{2}$. The only ways the parity of crossing between $e$ and another edge $f$ can change is by $e$ moving over an endpoint $v$ of $f$-corresponding to an $(e, v)$-move, or by the consecutive ends of $e$ and $f$ swapping order at a shared vertex - corresponding to a rotation swap.

\footnotetext{
${ }^{5}$ The slightly weaker result that this result is true if one restricts oneself to drawings in which adjacent edges do not cross, is already contained in Levow [17].
} 
Lemma 1.12 simplifies reasoning about drawings, by turning it into an algebraic problem. Our first use of the lemma is to establish a strengthening of Kleitman's observation. Let a principal $k$-cycle of a subdivision of a graph $G$ be a cycle that contracts to a $k$-cycle of $G$.

Lemma 1.13. Given a drawing of a subdivision of $K_{3,3}$, the number of principal 4-cycles with an odd number of independent self-crossings is odd.

Proof. The claim is true for the standard straight-line drawings of $K_{3,3}$ and its subdivisions, so we only have to show that the number of principal 4-cycles with an odd number of independent self-crossings does not change under $(e, v)$-moves.

First observe that an edge and a vertex in a $K_{3,3}$ always determine an even number of 4-cycles (namely 2 or 4 ) that use the edge and the vertex; on the other hand two disjoint edges determine a unique 4-cycle.

Fix a drawing of a $K_{3,3}$-subdivision, and select any vertex $v$ and edge $e$ of the graph. If $v$ is a degree- 2 vertex in a subdivided $K_{3,3}$ edge $f$, and $e$ occurs in the subdivision of a $K_{3,3}$-edge that is not adjacent to $f$, then the two edges incident to $v$ cannot be adjacent to $e$, so the $(e, v)$-move flips the parity of crossing of $e$ with both edges incident to $v$. Since either both or neither of those two edges belong to a cycle, the number of independent self-crossings along any cycle cannot change in this case.

Otherwise, $v$ is a degree- 2 vertex in the same subdivided edge that $e$ belongs to, or $v$ is a vertex of the original $K_{5}$. In either case, by the observation, an $(e, v)$-move affects an even number of principal 4-cycles (by either changing the parity of independent self-crossings of all of them or none of them), so the total number of principal 4-cycles with an odd number of independent self-crossings does not change parity.

As often, $K_{5}$ turns out to be the harder case.

Lemma 1.14. Any drawing of a $K_{5}$ contains a cycle with an odd number of independent self-crossings.

Proof. Let an $i$-odd pair be a pair of independent edges that crosses an odd number of times. First note that in any drawing of a $K_{5}$ the number of i-odd pairs is odd. This is true for the standard (convex) straight-line drawing of $K_{5}$, which contains five i-odd pairs; so it is sufficient to show that the parity of i-odd pairs does not change under $(e, v)$-moves; but this is clear, since any $(e, v)$-move either does not change the parity of crossings between independent edges at all (if $v$ is an endpoint of $e$ ), or changes the parity of crossing between $e$ and each of the two edges incident to $v$ that are not adjacent to $e$. In either case, the parity of i-odd pairs does not change.

Now assume, for a contradiction, that $K_{5}$ can be drawn so that every cycle has an even number of independent self-crossings. Every 4-cycle is made up of two independent pairs of edges that, by assumption, must have the same parity of crossing. But then the three pairs of independent edges that make up a $K_{4}$-subgraph must also all have the same parity of crossing. So each $K_{4}$ has 
either three or no i-odd pairs. Since the number of i-odd pairs in the drawing is odd, this implies that of the five $K_{4} \mathrm{~s}$ into which the independent pairs in $K_{5}$ can be partitioned, an odd number of them will contain three i-odd pairs. Now consider any $C_{5}$; it shares exactly one i-odd pair with each of the five $K_{4} \mathrm{~s}$ so it has an odd number of independent self-crossings, contradicting the assumption.

With Lemma 1.14 as the base case we are ready to deal with subdivisions of $K_{5}$.

Lemma 1.15. Any drawing of a subdivision of $K_{5}$ contains a cycle with an odd number of independent self-crossings.

Proof. For the purposes of this proof only, the parity of a cycle is the parity of the number of pairs of independent edges that belong to the cycle and cross oddly. Suppose then that there is a subdivision $G$ of $K_{5}$ which can be drawn so that all cycles have even parity.

By Lemma 1.14, $G$ cannot be $K_{5}$, so $G$ must contain a degree-2 vertex $v$. We show that in that case we can redraw $G$ so that $v$ can be contracted away without changing the parity of any cycle. This gives us an inductive proof of the lemma. For the redrawing it will be useful to understand the effects of $(e, v)$-moves on the parity of cycles: if $v$ is one of the endpoints of $e$, then an $(e, v)$-move has no effect on the parity of any pairs of edges crossing, and so it does not affect the parity of any cycles. If, on the other hand, $v$ is not a neighbor of either endpoint of $e$, then an $(e, v)$-move also has no effect on the parity of any cycle: each cycle has even degree at $v$, so an $(e, v)$-move will change the parity of crossing between $e$ and the two cycle edges incident to $v$, so the parity of the cycle does not change. Finally, we are in the case that there is an edge $f$ which shares one endpoint with $e$ and has $v$ as its other endpoint. Then an $(e, v)$-move will change the parity of every cycle that contains both $e$ and $f$ excepting, if it exists, a $C_{3}$ containing both $e$ and $f$; a $C_{3}$ always has even parity.

First suppose that $G$ contains a path $u v w x$ where $v$ and $w$ have degree 2 (so $u v w x$ is part of a subdivided edge). There are two possible obstacles to merging $v w$ and $w x$ into a single edge $v x$ without changing the parity of any cycle: some edge incident to $x$ crosses $v w$ oddly or $u v$ crosses $w x$ oddly. If $u v$ crosses $w x$ oddly, then perform an $(u v, x)$-move; since there cannot be an edge between $u$ and $x$, this does not change the parity of any cycle, and makes $u v$ cross $w x$ evenly. If there is an edge $f$ incident to $x$ that crosses $v w$ oddly, perform an $(f, v)$-move. As above, $f$ cannot have $u$ as an endpoint, so no cycle changes parity, and $f$ crosses $v w$ evenly. Hence, all edges incident to $v$ and $x$ (excepting $v w$ and $w x$ ) cross both $v w$ and $w x$ evenly, so we can replace $v w$ and $w x$ by a single edge $v x$ without changing the parity of any cycle.

If $G$ does not contain a path $u v w x$ as above and is not a $K_{5}$, it must contain a path $u v w$ so that both $u$ and $w$ have degree 4 and $v$ has degree 2 . If all edges (other than $u v$ and $v w$ ) incident to $u$ or $w$ cross both $u v$ and $v w$ evenly, then we can merge $u v$ and $v w$ into a single edge $u w$ without changing the parity of any cycle in $G$. Hence, there must be some edge $e$ incident to $u$ or $w$ that crosses 
$u v$ or $v w$ oddly. Without loss of generality, $e$ is incident to $u$. If $e$ crosses $u v$ oddly, then we can perform an $(e, u)$-move. This makes $e$ cross $u v$ evenly, and it does not change the parity of any cycle (since $e$ is incident to $u$ ). Hence, we can assume that $e$ crosses $v w$ oddly. Let $x$ be the other endpoint of $e$. If $x w$ is not an edge in $G$, then we can perform an $(e, w)$-move without changing the parity of any cycle, and making $e$ cross $v w$ evenly. Hence $f=x w$ must be an edge of $G$. But now $u v, v w, f, e$ form a 4 -cycle in $G$ which, by assumption, has an even number of independent crossings. However, there are only two independent pairs: $e, v w$ and $f, u v$. Since $e$ and $v w$ cross oddly, so must $f$ and $u v$. Now perform both an $(e, w)$ - and a $(f, u)$-move. This reduces the number of odd crossings along $u v w$ and does not change the parity of any cycle (each move by itself changes the parity of all cycles containing both $e$ and $f$; performed together, the parity of each cycle is unaffected). In summary, we can ensure that all edges incident to $u$ or $w$ cross both $u v$ and $v w$ evenly, so $u v$ and $v w$ can be merged into a single edge $u w$.

Since by Kuratowski's theorem every non-planar graph contains a subdivision of $K_{5}$ or $K_{3,3}$, Lemmas 1.13 and 1.15 imply the following theorem. The traditional strong Hanani-Tutte theorem is an immediate consequence.

Theorem 1.16. If a graph can be drawn so that all its cycles have an even number of independent self-crossings, then the graph is planar.

One might ask, whether it is possible to prove the strong Hanani-Tutte theorem or even Theorem 1.16 without taking recourse to Kuratowski's theorem? This is not an idle question as we will see in Section 1.4. For Theorem 1.16 we have to leave the question open, but there is an elementary proof of the Hanani-Tutte theorem - in the style of the proof of Theorem 1.3 - that does not use Kuratowski's theorem [34]. ${ }^{6}$ This, in turn, leads to the question of whether Kuratowski's theorem can be obtained from the Hanani-Tutte theorem. The answer is yes, as shown by van der Holst [51]. ${ }^{7}$

\subsection{Algorithmic and Algebraic Aspects}

While Kuratowski's theorem gives us a characterization of planar graphs, it does not directly lead to either an efficient planarity test or an efficient embedding technique for planar graphs. These problems were first addressed in the sixties, culminating in the linear-time algorithm by Hopcroft and Tarjan. The HananiTutte theorem offers an alternative algorithmic approach to planarity testing along two separate routes: one practical, the tree approach, based on work of

\footnotetext{
${ }^{6}$ Sarkaria [41] in 1991 claimed the same result. His proof contains several flaws: The redrawing suggested in his Figure 4 (page 82) introduces odd crossings between $\beta$ and edges that end between $\alpha$ and $\beta$. This not only changes the parity of crossings between two edges, but it may also introduce crossings with edges that have previously been cleared of crossings. Both problems can be dealt with, but, as far as we know, not by locally working with a single vertex. This is why we believe that Sarkaria's proof cannot be fixed along the lines described in his paper.

${ }^{7}$ Sarkaria [41] also claims this result, however we were not able to verify it.
} 
de Fraysseix and Rosenstiehl [10] and the more theoretical algebraic approach, first suggested by $\mathrm{Wu}[56,57] .^{8}$

\subsubsection{Trémaux Orders}

A Trémaux tree is a (rooted) depth-first search tree of a graph; it defines a partial order on the vertices of the tree, a Trémaux order, with the root as the smallest element. In a Trémaux order any non-tree edge of a graph has two endpoints that are comparable. Given a spanning tree $T$ of a graph $G$, a $T$-embedding of $G$ is a drawing of $G$ in which the edges of $T$ are crossing-free; let $T[e]$ denote the unique path in $T$ that connects the endpoints of $e$. Liu established the following characterization of planarity [10].

Theorem 1.17 (Trémaux Crossing Theorem (Liu)). If $T$ is a Trémaux tree of a non-planar graph $G$, then any $T$-embedding of $G$ contains two edges $e$ and $f$ that cross oddly, and so that $T[e]$ and $T[f]$ have an edge in common.

The Trémaux Crossing Theorem follows from the strong Hanani-Tutte theorem: in a $T$-embedding there are two independent edges $e$ and $f$ that cross oddly by HananiTutte; but then $T[e]$ and $T[f]$ must share an edge: if they only shared a vertex, the endpoints of $e$ and $f$ together with the shared vertex do not form a Trémaux order.

The Trémaux Crossing Theorem is at the root of de Fraysseix-Rosenstiehl's planarity criterion which has been used to justify the correctness of linear time planarity algorithms including Hopcroft-Tarjan and the Left-Right algorithm of de Fraysseix-Rosenstiehl [10].

\subsubsection{Algebraic Characterizations of Planarity}

Call a drawing of a graph $i$-even if all pairs of independent edges cross evenly. To find a planar embedding of a graph, it is enough to, $(i)$, find an i-even drawing of the graph (if this fails, the graph is not planar), and, $(i i)$, convert the i-even drawing into a planar drawing.

For step $(i)$ we can exploit the algebraic characterization of planarity suggested by the Hanani-Tutte theorem. For a given graph $G$, let $U(G)$ be the $\mathrm{GF}(2)$-vector space over the basis $[e, f]$, where $e$ and $f$ range over all independent pairs of edges of $G$ and $e<f$ in some ordering of the edges; to simplify notation, we allow $[f, e]$ for $[e, f]$ and let $[e, f]=0$ if $e$ and $f$ are not independent.

Consider a drawing $D$ of $G=(V, E)$. With $D$ we associate the vector

$$
\mathbf{x}_{D}:=\sum_{e<f}\left(\operatorname{cr}_{D}(e, f) \bmod 2\right)[e, f]
$$

where $\operatorname{cr}_{D}(e, f)$ is the number of crossings between $e$ and $f$ in $D$. Let $X(G):=$ $\left\{\mathbf{x}_{D}: D\right.$ is a drawing of $\left.G\right\}$. Also, we define $\mathbf{w}_{e, v}:=\sum_{f=(u, v)}[e, f]$ (this vector corresponds to the effect of an $(e, v)$-move). Let $W(G)$ be the $\mathrm{GF}(2)$-vector space in $U(G)$ spanned by the $\mathbf{w}_{e, v}, e \in E, v \in V$.

\footnotetext{
${ }^{8} \mathrm{Wu}$ 's 1985 papers are translations of work originally published in the 1970s in Chinese.
} 
Theorem 1.18 (Van Kampen, Tutte, Wu, Levow). The difference between any two vectors in $X(G)$ lies in $W(G)$ and if $\mathbf{x} \in X(G)$ and $\mathbf{w} \in W(G)$, then $\mathbf{v}+\mathbf{w} \in X(G)$. In other words, $X(G)$ is a coset of $W(G)$ in $U(G)$.

Who proved this result? Van Kampen [54] proved one direction, $X(G)-X(G) \subseteq$ $W(G)$, but could only prove the other direction for higher dimensions; the first explicit statements are in $\mathrm{Wu}[56]$ and Levow [17]. Tutte essentially proved the same result over a different vector space, as we will see below; Levow was aware of Tutte's work, Wu was not.

By Theorem 1.18 planarity is equivalent to $\mathbf{0} \in X(G)$, which means that planarity can be phrased as system of linear equations over the field GF(2) which can be solved by Gaussian elimination in cubic time (even less). Unfortunately, the number of variables and equations is $\Theta\left(|E|^{2}\right)$, which yields a planarity algorithm running in worst-case time $O\left(|E|^{6}\right)$; it is not clear whether the structure of the problem can be used to obtain a practical algorithm.

Let $\|\mathbf{x}\|_{1}$ denote the 1-norm of $\mathbf{x}$, that is, the sum of the absolute values of the entries of $\mathbf{x}$. Then $\min _{\mathbf{x} \in X(G)}\|\mathbf{x}\|_{1}$ is known as the independent odd crossing number of $G$ written as iocr $(G)$ (see Section 3.4 for more on crossing numbers). Thus, computing iocr $(G)$ can be expressed as a minimization problem over a vector space. More precisely, let $\mathbf{s} \in X(G)$ be an arbitrary vector (for example, position the vertices of $G$ on the boundary of a disk in arbitrary order and consider the resulting straight-line drawing). Then $\operatorname{iocr}(G)$ is the minimum of $\|\mathbf{s}+\mathbf{x}\|_{1}$ over all $\mathbf{x} \in W(G)$. In other words, we are looking for $\mathbf{x} \in W(G)$ that is closest, in the 1-norm, to $\mathbf{s}$. This is a special case of the nearest vector problem, which is NP-hard to approximate to within any constant [3]. Hence, the algebraic approach does not seem to offer any help in the efficient computation or approximation of crossing numbers.

We have counted crossings along edges modulo 2, corresponding to the traditional Hanani-Tutte theorem; however, there is another way to count crossings that might be worth exploring, and that goes back at least as far as Whitney's 1944 paper [55], though Flores already hints at the possibility [20, 62 . Kolloquium $\S 12]$; it's first explicitly worked out by Tutte. Form $U_{\mathbb{Z}}(G)$ like $U(G)$, but as a $\mathbb{Z}$-vector space. If we orient all the edges in $G$, we can assign +1 and -1 to each crossing between two edges depending on the direction of the crossing; let $\operatorname{acr}_{D}(e, f)$ be the sum of these values along $e$ and $f$, and $\mathbf{a}_{D}:=\sum_{e<f} \operatorname{acr}_{D}(e, f)[e, f]$, and $A(G):=\left\{\mathbf{a}_{D}: D\right.$ is a drawing of $\left.G\right\}$. For an $(e, v)$-move we now have two vectors $\mathbf{w}_{e, v}$ depending on the direction in which we pull $e$ over $v$ (and the entries are +1 and -1 depending on the direction of crossing). Without spelling out the details, let $W_{\mathbb{Z}}(G)$ be the $\mathbb{Z}$-vector space generated by all these vectors.

Theorem 1.19 (Tutte). $A(G)$ is a coset of $W_{\mathbb{Z}}(G)$ in $U_{\mathbb{Z}}(G)$.

As above, planarity is equivalent to $\mathbf{0} \in A(G)$. Tutte also showed that if $\mathbf{x} \in A(G)$, then $2 \mathbf{x} \in W_{\mathbb{Z}}(G) .{ }^{9}$

\footnotetext{
${ }^{9}$ In his terminology: "A crossing chain is half a cross-coboundary."
} 
As with $X(G)$, we can make $A(G)$ the basis of a crossing number definition: $\min _{\mathbf{x} \in A(G)}\|\mathbf{x}\|_{1}$ is the independent algebraic crossing number of $G, \operatorname{or} \operatorname{iacr}(G)$, for short. On the question of whether iacr $=\mathrm{cr}$, where $\mathrm{cr}$ is the traditional crossing number (see Section 3.4), Levow [17] writes "it seems reasonable to hope that equality holds for all graphs"; as we will see in Section 3.4 this is not the case, the two notions of crossing numbers differ. Interestingly, Whitney came close to asking the same question 30 years earlier [55]. Levow continues "whether or not equality holds, the algebraic setting may be useful in helping to compute crossing numbers, for it leads to a lower bound for the crossing number given in terms of the solution to an integer or Boolean minimization problem."

Step (ii) requires an effective version of the strong Hanani-Tutte theorem. From that perspective all the proofs based on Kuratowski's theorem fail. The first proof of strong Hanani-Tutte that does not appeal to Kuratowski's theorem and constructs an embedding starting with an i-even drawing is from [34] (a straightforward implementation of the algorithm will run in quadratic time, better bounds might be possible). The approach has a flavor similar to the proof shown in Theorem 1.3. For a variant see Theorem 3.14, for a strengthening, Theorem 3.17 in Section 3.4. ${ }^{10}$

As an immediate consequence of steps $(i)$ and $(i i)$ we get that planarity testing can be performed, and the planar graph embedded, in time $O\left(|E|^{6}\right)$, so the algebraic approach, at least at this point, does not seem to offer any algorithmic advantages over the graph-theoretical approach. However, on the theoretical side, the algebraic point of view has led to interesting research, including several recent papers by van der Holst giving purely algebraic characterizations of planarity, outerplanarity, and linkless embeddability [51, 52].

\section{Surfaces}

Different from many other planarity criteria - such as Kuratowski or MacLane'sHanani-Tutte can easily be restated for arbitrary surfaces. ${ }^{11}$ Take the weak Hanani-Tutte theorem:

Theorem 2.1 (Weak Hanani-Tutte for Surfaces $[7,36]$ ). If a graph can be drawn in a surface $S$ so that every pair of edges crosses an even number of times, then the graph can be embedded in $S$ without changing the embedding scheme. ${ }^{12}$

\footnotetext{
${ }^{10}$ We note that there were two previous claims for algorithms solving (ii). Wu extends the system of linear equations by a set of quadratic equations whose solution will describe an embedding. However, solving quadratic systems of equations is NP-complete, so Wu's approach does not lead to an efficient solution. Sarkaria [41] claims that there is a "onedimensional version of Whitney's trick by means of which any graph $[G$ which has an i-even drawing] can be, step by step embedded in $\mathbb{R}^{2}$." Unfortunately, his version of the Whitney trick for $n=1$ is fatally flawed as explained in an earlier footnote.

${ }^{11}$ Both Kuratowski and MacLane can be restated for arbitrary surfaces, but in the case of Kuratowski we do not know the list of excluded topological minors except for the plane and the projective plane [21], and in the case of MacLane's criterion the generalization is far from obvious [5].

${ }^{12}$ Embedding schemes generalize the notion of rotation system to arbitrary surfaces, includ-
} 
For orientable surfaces this was established by Cairns and Nikolayevsky [7] using homology theory; the result can also be established using a geometric proof which also works for non-orientable surfaces as shown by Pelsmajer, Schaefer, and Stefankovič [36]. Cairns and Nikolayevsky established the slightly stronger Theorem 1.8 for orientable surfaces. The parity of crossing between two closed curves in a surface depends only on their isotopy classes (we assume the two curves have a finite number of crossings and don't touch at any point); for example, in the plane any two closed curves cross an even number of times, and any two generators of the torus will cross an odd number of times. Given two cycles $C_{1}$ and $C_{2}$ in a graph drawn in surface $S$, let $c_{1}$ and $c_{2}$ be two curves isotopic to the drawings of $C_{1}$ and $C_{2}$ that cross finitely and don't touch. Let $\Omega_{S}\left(C_{1}, C_{2}\right)$ denote the parity of crossing between $c_{1}$ and $c_{2}$. We use $\sigma_{S}\left(C_{1}, C_{2}\right)$ for the notion of crossing number defined at the beginning of Section 1.2 generalized to surface $S$.

Theorem 2.2 (Cairns, Nikolayevsky [7]). If a graph can be drawn in a surface $S$ so that $\sigma_{S}\left(C_{1}, C_{2}\right) \equiv \Omega_{S}\left(C_{1}, C_{2}\right)$ mod 2 for every two cycles in the graph, then the graph can be embedded in $S$ without changing the embedding scheme.

In the plane, $\Omega\left(C_{1}, C_{2}\right)=0$ for all pairs of cycles and so the planar version, Theorem 1.8, is a special case. The proof we sketched of Theorem 1.8 generalizes to arbitrary surfaces, including non-orientable surfaces: The parity of $\sigma\left(C_{1}, C_{2}\right)$ is not affected by contractions of edges, so we can contract the graph to a single vertex with loops; now the number of crossing between two loops $e$ and $f$ equals $\sigma_{S}(e, f)+$ $\Omega_{S}(e, f) \equiv 0 \bmod 2$, so $e$ and $f$ cross an even number of times. By Theorem 2.1 that graph can be embedded without changing its embedding scheme.

The Loebl-Masbaum result, Theorem 1.3 also generalizes to arbitrary surfaces.

Theorem 2.3. If a graph can be drawn in surface $S$ so that every even subgraph has an even number of self-crossings, then $G$ can be embedded in $S$ without changing the embedding scheme.

Proof. The proof of Theorem 1.3 does not use the fact that the ambient surface is a plane until it deals with the one-vertex case. However, in the case of a single vertex the assumption of the theorem implies that any two loops cross evenly. This allows us to apply Theorem 2.1 (which, by its proof, is true for graphs with loops) to redraw the one-vertex graph without crossings and without changing the embedding scheme.

Similarly, a closer look at the proof of Theorem 1.4 shows that for 2-connected graphs it does not use planarity at all, but only relied on the weak Hanani-Tutte theorem for the plane. Since we just saw that that theorem can be lifted to arbitrary surfaces, the cycle version of weak Hanani-Tutte is true for arbitrary surfaces:

ing non-orientable ones. We do not include a formal definition, but refer the reader to [21] or [36] for details. 
Theorem 2.4. If a 2-connected graph can be drawn in surface $S$ so that every cycle has an even number of self-crossings, then the graph can be embedded in $S$ without changing the embedding scheme.

The result fails if the graph is not 2-connected; on the torus, for example, we can take two $K_{7}$ and overlap them in one vertex. Each $K_{7}$ by itself can be embedded on the torus without self-crossings, so all cycles in the drawing are free of self-crossings.

The planarity criterion by Lovász, Pach, and Szegedy, Theorem 1.9, does not seem to generalize in a straight-forward manner. The notion of converters seems too closely bound to the plane, but a careful analysis of the proof might yield an equivalent form for higher-order surfaces, at least for orientable surfaces.

The story of strong versions of Hanani-Tutte is, unfortunately, much shorter. We currently only know that strong Hanani-Tutte is true on the projective plane.

Theorem 2.5 (Pelsmajer, Schaefer, Stasi [32]). If a graph can be drawn in the projective plane so that every pair of independent edges crosses an even number of times, then the graph can be embedded in the projective plane.

The proof of Theorem 2.5 relies on the excluded minors for embeddability in the projective plane, so while some of ideas of the proof might be useful, it will not guide the way to establishing the Hanani-Tutte theorem for other surfaces, like the Klein bottle or the torus (for which the list of excluded minors is not known). It also means, that, at least with its present proof, Theorem 2.5 does not lead to an algorithm for embeddability in the projective plane. ${ }^{13}$

We know that in the plane every graph which can be drawn so that all its cycles have an even number of independent self-crossings is planar (Theorem 1.16). The example after Theorem 2.4 shows that on surfaces other than the plane we need to require 2 -connectedness.

Conjecture 2.6. If a 2-connected graph can be drawn in the projective plane so that all its cycles have an even number of independent self-crossings, then the graph can be embedded in the projective plane.

If all edges in a graph are independently even, all cycles in the graph have an even number of independent self-crossings. Therefore Conjecture 2.6 implies the strong Hanani-Tutte theorem on the projective plane; analogously, a version of Conjecture 2.6 for surface $S$ implies strong Hanani-Tutte of surface $S$. The reverse implication is not clear.

\footnotetext{
${ }^{13}$ In the terminology of Section 1.4, step (ii) fails. However, step (i) also seems to fail for the projective plane: the natural way of adding the cross-cap into the system of equations will lead to a quadratic system which, in general, is NP-complete to solve. Levow [17] shows how to extend Tutte's algebraic characterization of planarity to arbitrary surfaces.
} 


\section{Applications of Hanani-Tutte}

We survey some of the applications of Hanani-Tutte, both weak and strong; we do not claim completeness; for example, we do not pursue the alternative history of Hanani-Tutte in the literature of algebraic topology.

\subsection{Arrangements of Geometric Objects}

A collection of pseudo-disks is a collection of simply connected regions (bounded by simple, closed curves) so that the boundary curves of any two regions intersect at most twice. In their extension of a point-selection result from disks to pseudodisks, Smorodinsky and Sharir established the following theorem:

Theorem 3.1 (Smorodinsky, Sharir [45]). Let $P$ be a collection of $n$ points and $C$ a collection of $m$ pseudo-disks in the plane so that the boundary of every pseudo-disk passes through a distinct pair of points in $P$ and so that no pseudodisk contains a point of $P$ in its interior. Then $m \leq 3 n-6$.

To see that the theorem is true, construct a multi-graph on the vertices of $P$ with edges formed by the two boundary arcs of each pseudo-disk (formed by the two points of $P$ on the boundary). We argue that any two independent edges $e$ and $f$ of the graph cross an even number of times; suppose, for a contradiction, that $e$ and $f$ cross oddly. Since $e$ and $f$ are independent, they belong to two different pseudo-disks bounded by $e, e^{\prime}$ and $f, f^{\prime}$. Since $e$ and $f$ cross oddly, they must cross once (being part of the boundaries of two pseudo-disks, they can cross at most twice). If $e^{\prime}$ also crosses $f$, then neither can cross $f$ or $f^{\prime}$, so the two endpoints of $e$ are on different sides of the pseudo-circle formed by $f, f^{\prime}$, which is a contradiction, so we conclude that $e^{\prime}$ does not cross $f$. But then the endpoints of $f$ are on opposite sides of $e, e^{\prime}$, again a contradiction. Hence, any two independent edges cross evenly, and, thus, by Hanani-Tutte, the multigraph is planar. By construction, each edge in the multi-graph is doubled, so we conclude that $m \leq 3 n-6$. $^{14}$

The proof needs the strong Hanani-Tutte theorem, since adjacent edges might very well cross oddly in the setting of Theorem 3.1. This makes generalizations of Theorem 3.1 to other surfaces hard, since we do not have strong Hanani-Tutte for arbitrary surfaces; however, we can extend Theorem 3.1 to the projective plane: Note that apart from the application of Hanani-Tutte, the proof of Theorem 3.1 works for arbitrary surfaces, since the union of two pseudo-disks (even in surfaces other than the plane) is a planar region, so the argument can proceed as is; the only difference is that we need to replace the Euler bound on $m$ by the corresponding bound for the given surface; in the case of the projective plane, the proof outline above, combined with Theorem 2.5 establishes the following result:

Theorem 3.2. Let $P$ be a collection of $n$ points and $C$ a collection of $m$ pseudodisks in the projective plane so that the boundary of every pseudo-disk passes through a distinct pair of points in $P$ and so that no pseudo-disk contains a point of $P$ in its interior. Then $m \leq 3 n-3$.

\footnotetext{
${ }^{14}$ The argument closely follows the proof given in [45].
} 
To generalize Theorem 3.1 to arbitrary surfaces we do not actually need the full strong Hanani-Tutte theorem, since we can assume that any pair of edges crosses at most twice. This suggests the following parameterized form of the Hanani-Tutte theorem for arbitrary surfaces:

Conjecture 3.3. If a graph can be drawn in a surface $S$ so that any pair of independent edges crosses evenly and every pair of edges crosses at most $t$ times, then the graph can be embedded in the surface.

To establish Theorem 3.1 for surface $S$ (with adjusted Euler bound), it would be sufficient to prove the conjecture for $t=2$, but even $t=1$ does not appear to be obvious.

The idea of using Hanani-Tutte with unions of objects seems to have been first used by Pach and Sharir [25] in a new proof of an earlier result of Whitesides and Zhao. Call a collection of simply connected regions $k$-admissible if no region disconnects another, the boundaries of regions don't touch and they cross at most $k$ times (for example, collections of pseudo-disks that don't touch are 2-admissible).

Theorem 3.4 (Whitesides, Zhao). The boundary of the union of a $k$-admissible family of size $n \geq 3$ contains at most $k(3 n-6)$ arcs.

As in the case of Theorem 3.1, the proof does not rely on properties of the plane other than the application of the Hanani-Tutte theorem (establishing Conjecture 3.3 for $t=2 k$ would be sufficient), so the theorem can be established for the projective plane with a bound of $k(3 n-3)$; other surfaces remain open, as does the question of whether results based on Theorems 3.1 and 3.4 can be extended to surfaces other than the plane.

It appears that the Hanani-Tutte theorem can play a role in extending results about geometric disks to pseudo-disks; indeed, another example due to Buzaglo, Pinchasi, and Rote [6] concerns the Vapnik-Červonenkis-dimension of pseudodisks. The Vapnik-Červonenkis-dimension of a collection $\mathcal{C}$ of sets is the largest number of points such that every subset of the points can be obtained as an intersection of the set of points with a set in $\mathcal{C}$. It is well-known that the VapnikCervonenkis-dimension of disks is 3 (for any set of four points, there always is some subset of the points that cannot be obtained by intersecting the four points with a disk).

Theorem 3.5 (Buzaglo, Pinchasi, and Rote [6]). The Vapnik-Červonenkisdimension of any collection of pseudo-disks is at most 3.

The proof does not require the Hanani-Tutte theorem, but it is based on studying drawings of $K_{4}$ in which edges are allowed to cross evenly.

A collection of pseudo-parabolas is a collection of functions from $\mathbb{R} \mapsto \mathbb{R}$ so that any two functions cross twice or share one point of tangency (and no point lies on more than two pseudo-parabolas). Then the tangency graph in which each pseudo-parabola is represented by a vertex, and an edge represents tangency between two pseudo-parabolas is a biparite, planar graph and thus has at most 
$2 n-4$ edges, if $n \geq 3$ is the number of pseudo-parabolas. This result from [1] is based on strong Hanani-Tutte and it has several interesting consequences, for example, that the number of empty bigons (or lenses) in a collection of pairwise intersecting pseudo-circles is linear in the number of pseudo-circles [1], also see the exposition in [26, Section 5.2]. Ezra and Sharir [12] use the same approach to bound the complexity of the lower envelope of $n$ functions in $\mathbb{R}^{3}$ (under certain conditions), a much more complicated situation.

\section{$3.2 \quad$ Excluded Subgraphs}

Traditionally, $\operatorname{ex}(n, G)$ is the largest number of edges of a (simple) graph on $n$ vertices without a subgraph isomorphic to $G$; in topological graph theory the corresponding notion is $\operatorname{ex}_{\mathrm{cr}}(n, G)$, asking for the largest number of edges in a topological graph that contains no self-intersecting $G$ (equivalently: all copies of $G$ are crossing free). Pinchasi and Radoičić introduced a parity version of this they called $\operatorname{ex}_{\mathrm{ocr}}(n, G)$ which is the largest number of edges of a topological graph on $n$ vertices for which every two edges in the same copy of $G$ cross evenly [40]. In the same spirit we can define $\operatorname{ex}_{i o c r}(n, G)$ in which we only require independent edges of $G$ to cross evenly (the reasons for the names will become clear in Section 3.4 on crossing numbers). By the definition we have $\operatorname{ex}(n, G) \leq \operatorname{ex}_{\mathrm{cr}}(n, G) \leq \operatorname{ex}_{\mathrm{ocr}}(n, G) \leq \operatorname{ex}_{i o c r}(n, G)$.

Let us consider two trivial cases. If $G=P_{3}$, the path of length 3 , then $\operatorname{ex}\left(n, P_{3}\right)=$ $\lfloor n / 2\rfloor$, the size of a largest matching on $n$ vertices. On the other hand, in a convex straight-line drawing of $K_{n}$ no two adjacent edges cross, so ex $\operatorname{cr}_{\mathrm{cr}}\left(n, P_{3}\right)=\operatorname{ex}_{\mathrm{ocr}}\left(n, P_{3}\right)=$ $\operatorname{ex}_{i o c r}\left(n, P_{3}\right)=\left(\begin{array}{c}n \\ 2\end{array}\right)$; for the same reason, $\operatorname{ex}_{\mathrm{cr}}\left(n, K_{1, m}\right)=\operatorname{ex}_{\mathrm{ocr}}\left(n, K_{1, m}\right)=\operatorname{ex}_{i o c r}\left(n, K_{1, m}\right)=$ $\left(\begin{array}{c}n \\ 2\end{array}\right)$ so stars are not of interest. If $G=2 K_{2}$, two independent edges, then $\operatorname{ex}\left(n, 2 K_{2}\right)=$ $n-1$, for $n \geq 4$, and $\operatorname{ex}_{\mathrm{cr}}\left(n, 2 K_{2}\right) \leq \operatorname{ex}_{\mathrm{ocr}}\left(n, 2 K_{2}\right) \leq \operatorname{ex}_{i o c r}\left(n, 2 K_{2}\right)=3 n-6$, using the strong Hanani-Tutte theorem. From these examples, it is clear that ex differs from all the other variants, but we do not know of any examples separating $\mathrm{ex}_{\mathrm{cr}}, \mathrm{ex}_{\mathrm{ocr}}$ and $\mathrm{ex}_{\text {iocr }}$.

As Pach, Pinchasi, Tardos and Tóth [23] point out any of these notions are only interesting for planar bipartite graphs $G$; if $G$ is non-planar, then $\operatorname{ex}(n, G)=\operatorname{ex}_{\mathrm{cr}}(n, G)=\operatorname{ex}_{\mathrm{ocr}}(n, G)=\operatorname{ex}_{i o c r}(n, G)$ (using the Hanani-Tutte theorem), and if $G$ is not bipartite, $\operatorname{ex}(n, G)$ is already $\Omega\left(n^{2}\right)$ so there are no interesting asymptotic results.

So the smallest interesting cases to consider are $P_{4}$ and $C_{4}$ and there are bounds for both. Pach, Pinchasi, Tardos and Tóth show that both $\operatorname{ex}_{\mathrm{cr}}\left(n, P_{4}\right)$ and $\operatorname{ex}_{i o c r}\left(n, P_{4}\right)$ are of order $\Theta\left(n^{3 / 2}\right)[23]$ compared to the traditional ex $\left(n, P_{4}\right)=$ $n$. Pinchasi and Radoičić show that $\operatorname{ex}_{\mathrm{ocr}}\left(n, C_{4}\right)=O\left(n^{8 / 5}\right)[40]$, while $\operatorname{ex}\left(n, C_{4}\right)=$ $\Theta\left(n^{3 / 2}\right)$ which also is the best current lower bound on $\operatorname{ex}_{\text {ocr }}\left(n, C_{4}\right)$. The $C_{4}$ problem is particularly interesting, since it has implications for the number of cuts needed to turn an arrangement of pseudo-parabolas into pseudo-segments [40].

Other variants of these problems are possible; for example, Pach, Pinchasi, Tardos and Tóth study the geometric version $\operatorname{ex}_{\mathrm{rcr}}\left(n, P_{4}\right)$ of $\operatorname{ex}_{\mathrm{cr}}\left(n, P_{4}\right)$ (in which all edges are line segments). For geometric versions, the $\mathrm{ex}_{\mathrm{cr}}, \mathrm{ex}_{\mathrm{ocr}}$ and $\mathrm{ex}_{i o c r}$ 
versions collapse. One can also consider $\operatorname{ex}_{c r(2)}(n, G)\left(\operatorname{ex}_{c r_{-}(2)}(n, G)\right)$ in which we require that every copy of $G$ in the topological graph has an even number of (independent) self-crossings. This has the flavor of Theorem 1.4 and the problems suggested in the subsequent remark. As far as we know, nothing is known about $\mathrm{ex}_{c r(2)}$ and $\mathrm{ex}_{c r_{-}(2)}$ or how they relate to $\mathrm{ex}_{\mathrm{ocr}}$ or $\mathrm{ex}_{i o c r}$.

\subsection{Thrackles}

John Conway has a penchant for asking simple questions that are hard to answer. His Devil and Angel problem had to wait more than 20 years for a solution, and his even older thrackle conjecture is still unsettled. It forcefully drives home the point how little we really know about drawings of graphs.

Conway defined a thrackle as a graph that can be drawn so that every pair of edges intersects exactly once. A common endpoint of two edges counts as an intersection, so if we rephrase this condition in terms of crossings it requires that the graph can be drawn so that every pair of independent edges crosses exactly once and adjacent edges do not cross.

Conway conjectured that the number of edges of a thrackle is at most the number of its vertices [4, Section 9.5]. While this conjecture is open, we do know that $|E(G)|=O(|V(G)|)$ for thrackles $G$; this was first shown by Lovász, Pach and Szegedy [19]. ${ }^{15}$ Their proof uses the notion of a generalized thrackle, a graph which can be drawn so that every two edges cross an odd number of times.

The traditional definition of a generalized thrackle requires that every two edges intersect an odd number of times. The two definitions are equivalent [36, Remark 4.2]: one can flip the rotation at each vertex changing the parity of crossing between any pair of adjacent edges to move back and forth between the two variants. For traditional thrackles this implies that an intersection-thrackle is always a crossing-thrackle, but the reverse is not true: $C_{4}$ is known not to have a (intersection)-thrackle drawing, but it can easily be drawn so that every pair of edges crosses exactly once. We are not aware of any research specifically on crossing-thrackles.

Theorem 3.6 (Lovász, Pach and Szegedy [19]). A bipartite graph is a generalized thrackle if and only if it is planar.

Let $G$ be a thrackle; split $V(G)$ into $V_{1}$ and $V_{2}$ so as to maximize the number of edges between $V_{1}$ and $V_{2}$. Then every vertex has at least as many neighbors in the other partition as it has in its own partition (otherwise we would move it to the other partition), so we can remove at most half the edges of $G$ to turn it into a bipartite graph $G^{\prime}$. By Theorem 1.9, $G^{\prime}$ is planar and thus has at most $2\left|V\left(G^{\prime}\right)\right|-4$ edges (using Euler); but then $|E(G)| \leq 4\left|V\left(G^{\prime}\right)\right|-8 \leq 4|V(G)|$, so $|E(G)|=O(|V(G)|)$ for thrackles $G$. In fact, this bound can be improved by sharpening the reasoning:

\footnotetext{
${ }^{15}$ The best current upper bound of, approximately, $|E|<1.428|V|$ is due to Fulek and Pach [13] using computational methods.
} 
Corollary 3.7 (Lovász, Pach and Szegedy [19]). If $G$ is a thrackle, then $|E(G)| \leq$ $2|V(G)|-3$.

We include a very simple proof of Theorem 3.6 based on a proof from [36]. Recall that an $(e, v)$-move pulls $e$ over $v$ changing the parity of crossing between $e$ and every edge incident to $v$ as shown in Figure 4 .

Proof of Theorem 3.6. Let $G$ be a bipartite graph and $U$ one of its partitions. Fix an order of the vertices in $U$ and say that e precedes $v$, if $e$ 's endpoint in $U$ precedes $v$ in the ordering of $U$. Now, for any pair $e \in E(G)$ and $u \in U$ such that $e$ precedes $u$ perform an $(e, u)$-move. The result of these moves is that the parity of any pair of independent edges changes, whereas the parity of any pair of adjacent edges remains unaffected. Reversing the rotation of every vertex in $U$ and redrawing the edges incident to it in a small neighborhood of the vertex changes the parity of every pair of adjacent edges. In summary, the parity of every pair of edges changed. This means that for a bipartite graph a drawing in which every pair of edges crosses oddly can be turned into a drawing in which every pair of edges crosses evenly and vice versa. This immediately implies that a planar bipartite graph is a generalized thrackle, and, in the reverse direction, that a generalized bipartite thrackle is planar by the weak HananiTutte theorem.

Cairns and Nikolayevsky showed that Theorem 1.9 remains true on any orientable surface. Indeed, the proof of Theorem 3.6 we just gave works for arbitrary surfaces if we replace the application of the weak Hanani-Tutte theorem for the plane with the version for an arbitrary surface.

Corollary 3.8 (Cairns, Nikolayevsky [7]). A bipartite graph is a generalized thrackle in a surface if and only if it can be embedded in that surface.

Cairns and Nikolayevsky managed to find a pleasant generalization of this result to non-bipartite graphs using the notion of a parity embedding which is is an embedding of a graph on a non-orientable surface so that even cycles are two-sided curves and odd cycles are one-sided curves.

Theorem 3.9 (Cairns, Nikolayevsky [8]). G is a generalized thrackle on an orientable surface $S$ if and only if $G$ has a parity embedding on the (nonorientable) surface obtained by adding a crosscap to $S$.

A short proof-sketch of the easy direction: Given $G$ in $S$ push each edge across the new crosscap as shown in Figure 5. Every pair of edges will then cross evenly, and thus, by Theorem 2.1 be embeddable in the new surface.

This result can be extended to non-orientable surfaces using the notion of an $X$-parity embedding. For a non-orientable surface, let $X$ be a particular crosscap of the surface; an $X$-parity embedding is an embedding in which a cycle is odd if and only if it passes through $X$ an odd number of times. (If $S$ is orientable, then a parity embedding on $S+X$ is the same as an $X$-parity embedding, so Theorem 3.9 is subsumed.) 

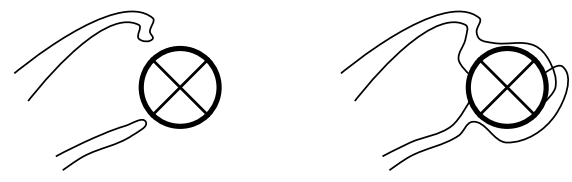

Figure 5: Pushing edges over the crosscap.

Theorem 3.10 (Pelsmajer, Schaefer, Štefankovič [36]). $G$ is a generalized thrackle on a surface $S$ if and only if $G$ has an $X$-parity embedding on the surface obtained by adding a crosscap $X$ to $S$, with the same embedding scheme. In that case, we can assume that every edge passes through $X$ an odd number of times.

As a consequence, we can recover a result shown by Perlstein and Pinchasi in their study of Vázsonyi's conjecture [39]. A centrally symmetric $S^{2}$-lifting of a graph $G$ is a bipartite graph $G^{\prime}$ embedded on the sphere so that $G^{\prime}$ is centrally symmetric and every vertex of $G$ corresponds to two antipodal points of $G^{\prime}$ that belong to different partitions of $G^{\prime}$ and every edge of $G$ corresponds to two edges of $G^{\prime}$ so that the endpoints of each edge belong to different partitions.

Theorem 3.11 (Perlstein, Pinchasi [39]). A graph is a generalized thrackle if and only if it has a centrally symmetric $S^{2}$-lifting.

Proof sketch: A centrally symmetric $S^{2}$-lifting is really a double-cover of an embedding of $G$ in the projective plane, so one direction is obvious. In the other direction, Theorem 3.10 tells us that a generalized thrackle can be embedded in the projective plane so that every edge crosses through the crosscap an odd number of times. If we think of the projective plane as a disk with the crosscap as its boundary, then the natural $S^{2}$-double-cover of this embedding is a centrally symmetric $S^{2}$-lifting.

A geometric graph is a graph with a straight-line embedding. With Theorem 3.11 Perlstein and Pinchasi are able to show that every geometric graph in $\mathbb{R}^{3}$ in which every two edges are strongly avoiding - they can be projected onto some 2-dimensional plane so that they belong to two distinct rays that form a non-acute angle between them - is a generalized thrackle (in the plane).

\subsection{Crossing Numbers}

The crossing number, $\operatorname{cr}(G)$, of a graph $G$ is the smallest number of crossings necessary to draw the graph in the plane. We do not allow edges to pass through vertices or more than two edges to cross in a point.

The Hanani-Tutte theorem has been closely linked to the study of the crossing number and many of its variants. Kleitman's parity result, for example, was part of his proof that Zarankiewicz's conjecture holds for the crossing numbers of $K_{5, n}$ and 
$K_{6, n}$. Tutte's paper tried to establish an algebraic theory of crossing numbers, see Section 1.4 for details on Tutte's approach.

Do we really need to count all the crossings? The (weak) Hanani-Tutte theorem seems to suggest that it should be sufficient to count crossings only modulo 2: let $\operatorname{ocr}(G)$, the odd crossing number of $G$ be the smallest number of pairs of edges that cross oddly in a drawing of $G$ [29]. The odd crossing number in many ways behaves like the standard crossing number, for example, the famous crossing lemma, $\operatorname{cr}(G) \geq 1 / 64|E(G)|^{3} /|V(G)|^{2}$, remains true for the odd crossing number, with the original proof, though some recent strengthening of the constant factor apparently do not carry over to the odd crossing number [24].

The weak Hanani-Tutte theorem can now be stated as saying that $\operatorname{ocr}(G)=0$ implies that $\operatorname{cr}(G)=0$. This might suggest that $\operatorname{ocr}(G)=\operatorname{cr}(G)$ for all $G$, but equality does not hold between the two crossing numbers: One can construct a graph for which $\operatorname{ocr}(G)<\operatorname{cr}(G) \leq 10[35,47]^{16}$. However, it is true that $\operatorname{ocr}(G)=\operatorname{cr}(G)$ as long as $\operatorname{ocr}(G) \leq 3$ [34], that is, if a graph can be drawn so that at most $k \leq 3$ edges cross oddly, then the graph can be drawn with at most $k$ crossings. To establish a result like this, one must be able to remove crossings along edges that are not involved in odd crossings. The first such "removing crossings" result is due to Pach and Tóth.

Lemma 3.12 (Pach, Tóth [29]). If $D$ is a drawing of $G$ in the plane, and $E_{0}$ is the set of even edges in $D$, then $G$ can be drawn in the plane so that no edge in $E_{0}$ is involved in any crossings.

Lemma 3.12 implies that cr cannot be arbitrary larger than ocr (clear all even edges of crossings using the lemma and then draw the remaining edges in their faces so they cross each other at most once), resulting in the pairwise crossing of at most $2 \operatorname{ocr}(G)$ edges. This was observed by Pach and Tóth.

Corollary 3.13 (Pach, Tóth $[29]) . \operatorname{cr}(G) \leq\left(\begin{array}{c}2 \operatorname{ocr}(G) \\ 2\end{array}\right)$.

Lemma 3.12 has the disadvantage that it may introduce new odd pairs, that is, pairs of edges that cross oddly, so it cannot be used to show, for example, that $\operatorname{ocr}(G)=\operatorname{cr}(G)$ for small values. This issue is addressed in the following variant.

Lemma 3.14 (Pelsmajer, Schaefer, Štefankovič [34]). If $D$ is a drawing of $G$ in the plane, and $E_{0}$ is the set of even edges in $D$, then $G$ can be drawn in the plane so that no edge in $E_{0}$ is involved in any crossings and there are no new pairs of edges that cross an odd number of times.

Lemma 3.14 can be used to prove the strong Hanani-Tutte theorem without appealing to Kuratowski's theorem: Pick a cycle in the graph, make its edges even (since there are no odd independent crossings this can be done by locally modifying rotations of vertices on the cycle), use Lemma 3.14 to remove crossings with the cycle, and

\footnotetext{
${ }^{16}$ The original separation is from [35]; Tóth's approach leads to a separation of $\operatorname{ocr}(G)$ and $\operatorname{cr}(G)$ with $\operatorname{cr}(G) \leq 10[48]$.
} 
induct. The induction has to be set up carefully. This approach yields an effective procedure for constructing the embedding from the original drawing.

Lemma 3.14 should be useful as a first step in improving the upper bound of Corollary 3.13. The general feeling is that $\operatorname{cr}(G) \leq O(\operatorname{ocr}(G))$, but there is no hard evidence for this. Lemma 3.14 unfortunately fails on surfaces other than the plane (there are counterexamples for projective plane and torus that show that the pairs of edges that cross oddly may have to change [36]). It is possible that a surface version of the lemma can be proved which only concludes that the odd crossing number of the drawing does not increase. Meanwhile, the following weaker version (which in the plane is the same as Pach-Tóth's result, Lemma 3.12) is true:

Lemma 3.15 (Pelsmajer, Schaefer, Štefankovič [36]). If $D$ is a drawing of a graph $G$ on some surface $S$, and $E_{0}$ is the set of even edges in $D$, then $G$ can be drawn in $S$ so that no edge in $E_{0}$ is involved in any crossings.

One concludes, as in the planar case, that $\mathrm{cr}_{S}$, the crossing number on surface $S$ is bounded in terms of $\operatorname{ocr}_{S}$, the odd crossing number on $S$ :

Corollary 3.16 (Pelsmajer, Schaefer, Štefankovič $[36]) \cdot \operatorname{cr}_{S}(G) \leq\left(\begin{array}{c}2 \operatorname{ocr}_{S}(G) \\ 2\end{array}\right)$ for any surface $S$, orientable or non-orientable.

The independent odd crossing number, $\operatorname{iocr}(G)$, is the smallest number of independent pairs of edges that cross in a drawing of $G$. The (strong) HananiTutte theorem translates into "iocr $(G)=0$ implies $\operatorname{cr}(G)=0$ ". Since, by definition, $\operatorname{iocr}(G) \leq \operatorname{ocr}(G) \leq \operatorname{cr}(G)$, we already know that equality does not hold between $\operatorname{iocr}(G)$ and $\operatorname{cr}(G)$ (as ocr and cr can be separated), however, it is entirely open whether $\operatorname{iocr}(G)=\operatorname{ocr}(G)$. One might again ask, whether $\operatorname{iocr}(G)=\operatorname{cr}(G)$ for small values and whether cr can be bounded in terms of iocr. The situation is more difficult than cr versus ocr, since the number of odd crossings in a drawing can be arbitrarily large even if iocr is bounded. However, we could recently establish the following redrawing result:

Lemma 3.17 (Pelsmajer, Schaefer, Štefankovič [37]). If $D$ is a drawing of a graph $G$ in the plane and $E_{0}$ is the set of independently even edges in $D$, then $G$ can be redrawn so that no edge in $E_{0}$ is involved in any crossings and every pair of edges crosses at most once.

As an immediate consequence one obtains, as earlier:

Corollary 3.18 (Pelsmajer, Schaefer, Štefankovič [37]) $\operatorname{cr}(G) \leq\left(\begin{array}{c}2 \operatorname{iocr}(G) \\ 2\end{array}\right)$.

It is open whether the conclusion of Lemma 3.17 can be strengthened to say that there are no new pairs of independent edges crossing oddly. The following result is another consequence of Lemma 3.17; the proof in this case is rather intricate though.

Corollary 3.19 (Pelsmajer, Schaefer, Štefankovič [37]). $\operatorname{iocr}(G)=\operatorname{cr}(G)$ for graphs $G$ with $\operatorname{iocr}(G) \leq 2$. 
We do not know whether any of the last three results hold for surfaces other than the plane.

We started with the odd and independent odd crossing numbers, since they are most closely related to the Hanani-Tutte results, however, there are two other crossing number variants worth mentioning in this context: pair and algebraic crossing number (the latter we saw before in Section 1.4). With this, our - still incomplete - list of basic crossing number variants becomes:

crossing number: $\operatorname{cr}(G)$, the smallest number of crossings in a drawing of $G$,

pair crossing number: $\operatorname{pcr}(G)$, the smallest number of pairs of edges crossing in a drawing of $G$,

algebraic crossing number: $\operatorname{acr}(G)$, orient all the edges in a drawing and distinguish between positive and negative crossings along an edge, counting them as +1 and -1 ; minimize the sum of the absolute values of these counts for each edge,

odd crossing number: $\operatorname{ocr}(G)$, the smallest number of pairs of edges crossing oddly in a drawing of $G$.

We can modify each of these notions by two rules, suggested by Pach and Tóth [28]:

"Rule +": restrict the drawings to drawings in which adjacent edges are not allowed to cross.

"Rule -": allow crossings of adjacent edges, but does not count them towards the crossing

We add + and - as a subscript to the crossing number to denote that we are following that particular rule. Rule + is inspired by the observation that crossing-number minimal drawings fulfill it, that is, $\mathrm{cr}=\mathrm{cr}_{+}$, but it is not clear whether this holds for any other crossing number variant. Of the twelve possible combinations of Rule + and Rule - with the four crossing numbers, these are the only two that are known to coincide. Rule - is what turns ocr into iocr, namely, ocr $_{-}=$iocr.

This leaves us with eleven, potentially different, notions of crossing number:

\begin{tabular}{l||l|l|l|l} 
Rule + & ocr $_{+}$ & acr $_{+}$ & pcr $_{+}$ & \multirow{2}{*}{$\mathrm{cr}$} \\
\hline & ocr & acr & pcr & \\
\hline Rule - & iocr $=$ ocr $_{-}$ & iacr $=$acr $_{-}$ & pcr $_{-}$ & cr \\
\hline
\end{tabular}

Little is known about the relationship between these crossing numbers. The variants are monotone in the sense that going from bottom to top in the table does not decrease the value and neither does going from left to right as long as we drop either the acr-column or the pcr-column. So $\operatorname{iocr}(G)$ is the smallest and $\operatorname{cr}(G)$ the largest value, but we do not know whether $\operatorname{acr}(G) \leq \operatorname{pcr}(G)$. We 
are aware of only two separations among all these variants: one can construct families of graphs for which $\operatorname{ocr}(G)<\lambda \operatorname{cr}(G)$ for some $\lambda<1$ (originally [35], improved $\lambda$ in [47]). The original examples realize $\operatorname{ocr}(G)<\operatorname{acr}(G)=\operatorname{pcr}(G)=$ $\operatorname{cr}(G)$ [35] and the new examples $\operatorname{ocr}(G)=\operatorname{acr}(G)<\operatorname{pcr}(G)=\operatorname{cr}(G)$ [47]. Combining these two types of examples, one can build a graph $G$ for which $\operatorname{ocr}(G)<\operatorname{acr}(G)<\operatorname{pcr}(G)$. We are not aware of any other separations. Corollary 3.18 shows that all the crossing number variants listed here are within a square of each other; for cr versus pcr this bound can be improved: Valtr [50] showed that $\operatorname{cr}(G)=O\left(\operatorname{pcr}^{2}(G) / \log \operatorname{pcr}(G)\right)$, which Tóth [47] improved to $\operatorname{cr}(G)=O\left(\operatorname{pcr}^{2}(G) / \log ^{2} \operatorname{pcr}(G)\right)$. Using a separator theorem for string graphs due to Pach and Fox, Tóth has recently been able to lower this bound to $\operatorname{cr}(G)=O\left(\operatorname{pcr}^{7 / 4}(G) / \log ^{3 / 2} \operatorname{pcr}(G)\right)[46]$. These are the only non-quadratic upper bounds between crossing numbers we are aware of.

We conjecture that $\mathrm{pcr}=\mathrm{cr}$ and $\mathrm{cr}_{-}=\mathrm{cr}$. Evidence for the first conjecture is purely computational: for two vertex multi-graphs with rotations it appears to be true, according to computer searches performed in connection with [35]. A first step towards the second conjecture is the proof that crossings with adjacent edges can be removed if they are the only crossings along an edge:

Theorem 3.20 (Schaefer [42]). If $D$ is a drawing of $G$ in the plane, and $E_{0}$ is the set of edges in $D$ that have no independent crossings, then $G$ can be drawn in the plane so that no edge in $E_{0}$ is involved in any crossings and there are no new independent crossings.

This brings us only slightly closer to proving $\mathrm{cr}_{-}=\mathrm{cr}$, but it is a first, necessary, step to showing even $\mathrm{cr}=O\left(\mathrm{cr}_{-}\right)$. The extent of our ignorance about $\mathrm{cr}_{-}$is captured in the following conjecture.

Conjecture 3.21. If a graph can be drawn on a surface $S$ so that no two independent edges cross, then the graph can be embedded in $S$.

By the strong Hanani-Tutte theorem we know that the conjecture is true for the plane and the projective plane. Beyond that we know nothing. So we can only agree with the first half of Tutte's sentiment that "crossings of adjacent edges are trivial, and easily got rid of" [49].

What about the computational complexity of all of these crossing numbers? Obviously, they are all NP-complete? Well, yes, and no. Most of them are NP-complete, but not always for obvious reasons. Garey and Johnson showed that the crossing number problem is NP-complete [14]. This proof also shows that pcr is NP-hard, but it does not imply that the problem lies in NP; that was established later in connection with the string graph problem [43]. The variant ocr is NP-complete as shown by Pach and Tóth [29]; NP-hardness is a modification of the Garey-Johnson proof and containment in NP relies on a refinement of Theorem 1.18 which takes into account the rotation system. For acr, Pach and Tóth's hardness proof for ocr still works and acr $\in \mathbf{N P}$, since $\operatorname{acr}(G) \leq k$ can be rephrased as an integer linear program (along the lines of Theorem 1.19). (This means that drawings of $G$ with $\operatorname{acr}(G) \leq k$ may require an exponential number of crossings, since this is the best bound known for integer linear programming. We leave it open whether this bound can be improved for acr.) 
For the Rule - variants, we know that iocr, pcr_ and cr - are NP-complete [38]. In all three cases, NP-hardness follows from showing that the underlying crossing number concept ocr, pcr and cr remains NP-hard if the graph is given with a rotation system. (This turns out to be highly non-trivial in the case of ocr.) All three problems lie in NP; for iocr this follows directly from Theorem 1.18, for $\mathrm{pcr}_{-}$and $\mathrm{cr}_{-}$the situation is more complicated, since there is no immediate bound on the number of crossings that do not count; using techniques from [43] and [44] the problems can be placed in NP (the upper bounds on the uncounted crossings are exponential). We do not know the complexity of iacr, though it is quite possible that the iocr-hardness proof can be adapted.

Finally, ocr + and pcr $_{+}$are NP-hard; we know that ocr and pcr remain hard if the rotation system of the graph is specified. So let $(G, R)$ be a graph with rotation system; from it construct $G^{\prime}$ by replacing each vertex $v$ of degree $d$ with a wheel $W_{d}$ and attach edges originally connected to $v$ to the $d$ outer vertices of $W_{d}$. Finally, replace edges of $W_{d}$ with multiple, parallel $P_{3}$ s to ensure that the $W_{d}$ are embedded. Then the pair or odd crossing number of $G$ with rotation $R$ is at most $k$ if and only if $\mathrm{pcr}_{+}$or $\mathrm{ocr}_{+}$of $G^{\prime}$ is at most $k$. Showing that ocr $_{+}$and $\mathrm{pcr}_{+}$lie in NP can be done using the approach from [43] and [44]. Again we leave open the complexity of the algebraic variant, $\mathrm{acr}_{+}$.

There are also results on the parameterized complexity of pcr and ocr [33].

\section{In Place of a Conclusion}

We have sprinkled open problems and conjectures liberally throughout the survey and there are many obvious questions one can ask (is there a Hanani-Tutte theorem for hypergraphs? For matroids?), so instead of reiterating this material, let us mention one more tempting direction that one can take the Hanani-Tutte theorem. We restricted ourselves to surfaces following the graph-theoretical tradition; the algebraic topology literature went a different route. The HananiTutte theorem is there known as a version of the Flores-van Kampen theorem, which has many generalizations and variants, typically in higher-dimensional spaces. Closer to our versions of the Hanani-Tutte theorem is a recent result of van der Holst and Pendavingh [53]: imagine a graph embedded in $\mathbb{R}^{3}$. An embedding is called flat if one can attach an open disk to each cycle of the graph so that the boundary of the disk is the cycle and the disk is disjoint from the graph. If a graph can be embedded in $\mathbb{R}^{3}$ so that each disk crosses each non-incident edge of the graph an even number of times, then the graph has a flat embedding. The proof uses methods from algebraic topology; can it be shown using an intuitive geometric argument?

\section{Acknowledgment}

I would like to thank Martin Loebl, Hein van der Holst and the anonymous referee for helpful comments. And Daniel and Michael for being there. Without them there would have been less to survey. 


\section{References}

[1] Pankaj K. Agarwal, Eran Nevo, János Pach, Rom Pinchasi, Micha Sharir, and Shakhar Smorodinsky. Lenses in arrangements of pseudo-circles and their applications. J. ACM, 51(2):139-186 (electronic), 2004.

[2] Dan Archdeacon and R. Bruce Richter. On the parity of crossing numbers. J. Graph Theory, 12(3):307-310, 1988.

[3] Sanjeev Arora, László Babai, Jacques Stern, and Z. Sweedyk. The hardness of approximate optima in lattices, codes, and systems of linear equations. $J$. Comput. System Sci., 54(2, part 2):317-331, 1997. 34th Annual Symposium on Foundations of Computer Science (Palo Alto, CA, 1993).

[4] Peter Brass, William Moser, and János Pach. Research Problems in Discrete Geometry. Springer, New York, 2005.

[5] Henning Bruhn and Reinhard Diestel. Maclane's theorem for arbitrary surfaces. Journal of Combinatorial Theory, Series B, 99(2):275 - 286, 2009.

[6] Sarit Buzaglo, Rom Pinchasi, and Günter Rote. Topological hyper-graphs. Unpublished manuscript, 2007.

[7] Grant Cairns and Yury Nikolayevsky. Bounds for generalized thrackles. Discrete Comput. Geom., 23(2):191-206, 2000.

[8] Grant Cairns and Yury Nikolayevsky. Generalized thrackle drawings of non-bipartite graphs. Discrete Comput. Geom., 41(1):119-134, 2009.

[9] Jakub Černý. Combinatorial and Computational Geometry. PhD thesis, Charles University, Prague, 2008.

[10] Hubert de Fraysseix and Pierre Rosenstiehl. A characterization of planar graphs by Trémaux orders. Combinatorica, 5(2):127-135, 1985.

[11] Peter Eades, Qing-Wen Feng, and Xuemin Lin. Straight-line drawing algorithms for hierarchical graphs and clustered graphs. In Graph drawing, volume 1190 of Lecture Notes in Comput. Sci., pages 113-128, London, UK, 1997. Springer.

[12] Esther Ezra and Micha Sharir. Lower envelopes of 3-intersecting surfaces in $\mathbb{R}^{3}$. Unpublished manuscript, 2007.

[13] Radoslav Fulek and János Pach. A computational approach to conway's thrackle conjecture. CoRR, abs/1002.3904, Feb 2010.

[14] Michael R. Garey and David S. Johnson. Crossing number is NP-complete. SIAM Journal on Algebraic and Discrete Methods, 4(3):312-316, 1983.

[15] Chaim Chojnacki (Haim Hanani). Über wesentlich unplättbare Kurven im drei-dimensionalen Raume. Fundamenta Mathematicae, 23:135-142, 1934. 
[16] Daniel J. Kleitman. A note on the parity of the number of crossings of a graph. J. Combinatorial Theory Ser. B, 21(1):88-89, 1976.

[17] Roy B. Levow. On Tutte's algebraic approach to the theory of crossing numbers. In Proceedings of the Third Southeastern Conference on Combinatorics, Graph Theory, and Computing (Florida Atlantic Univ., Boca Raton, Fla., 1972), pages 315-314, Boca Raton, Fla., 1972. Florida Atlantic Univ.

[18] Martin Loebl and Gregor Masbaum. On the optimality of the arf invariant formula for graph polynomials. CoRR, abs/0908.2925, 2009.

[19] Laszlo Lovász, János Pach, and Mario Szegedy. On Conway's thrackle conjecture. Discrete Comput. Geom., 18(4):369-376, 1997.

[20] Karl Menger. Ergebnisse eines mathematischen Kolloquiums. SpringerVerlag, Vienna, 1998.

[21] Bojan Mohar and Carsten Thomassen. Graphs on surfaces. Johns Hopkins Studies in the Mathematical Sciences. Johns Hopkins University Press, Baltimore, MD, 2001.

[22] Serguei Norine. Pfaffian graphs, T-joins and crossing numbers. Combinatorica, 28(1):89-98, 2008.

[23] János Pach, Rom Pinchasi, Gábor Tardos, and Géza Tóth. Geometric graphs with no self-intersecting path of length three. European J. Combin., 25(6):793-811, 2004.

[24] János Pach, Radoš Radoicić, Gábor Tardos, and Géza Tóth. Improving the crossing lemma by finding more crossings in sparse graphs: [extended abstract]. In $S C G$ '04: Proceedings of the twentieth annual symposium on Computational geometry, pages 68-75, New York, NY, USA, 2004. ACM.

[25] János Pach and Micha Sharir. On the boundary of the union of planar convex sets. Discrete Comput. Geom., 21(3):321-328, 1999.

[26] János Pach and Micha Sharir. Geometric incidences. In Towards a theory of geometric graphs, volume 342 of Contemp. Math., pages 185-223. Amer. Math. Soc., Providence, RI, 2004.

[27] János Pach and Micha Sharir. Combinatorial geometry and its algorithmic applications: The Alcalá lectures, volume 152 of Mathematical Surveys and Monographs. American Mathematical Society, Providence, RI, 2009.

[28] János Pach and Géza Tóth. Thirteen problems on crossing numbers. Geombinatorics, 9(4):194-207, 2000.

[29] János Pach and Géza Tóth. Which crossing number is it anyway? J. Combin. Theory Ser. B, 80(2):225-246, 2000. 
[30] János Pach and Géza Tóth. Monotone drawings of planar graphs. J. Graph Theory, 46(1):39-47, 2004.

[31] János Pach and Géza Tóth. Disjoint edges in topological graphs. In Combinatorial geometry and graph theory, volume 3330 of Lecture Notes in Comput. Sci., pages 133-140. Springer, Berlin, 2005.

[32] Michael J. Pelsmajer, Marcus Schaefer, and Despina Stasi. Strong HananiTutte on the projective plane. SIAM Journal on Discrete Mathematics, 23(3):1317-1323, 2009.

[33] Michael J. Pelsmajer, Marcus Schaefer, and Štefankovič. Crossing numbers and parameterized complexity. In Seok-Hee Hong, Takao Nishizeki, and Wu Quan, editors, Graph Drawing, volume 4875 of Lecture Notes in Computer Science, pages 31-36. Springer, 2007.

[34] Michael J. Pelsmajer, Marcus Schaefer, and Daniel Štefankovič. Removing even crossings. J. Combin. Theory Ser. B, 97(4):489-500, 2007.

[35] Michael J. Pelsmajer, Marcus Schaefer, and Daniel Štefankovič. Odd crossing number and crossing number are not the same. Discrete Comput. Geom., 39(1):442-454, 2008.

[36] Michael J. Pelsmajer, Marcus Schaefer, and Daniel Štefankovič. Removing even crossings on surfaces. European J. Combin., 30(7):1704-1717, 2009.

[37] Michael J. Pelsmajer, Marcus Schaefer, and Daniel Štefankovič. Removing independently even crossings. SIAM Journal on Discrete Mathematics, 24(2):379-393, 2010.

[38] Michael J. Pelsmajer, Marcus Schaefer, and Daniel Štefankovič. Crossing numbers of graphs with rotation systems. Algorithmica, 60:679-702, 2011. 10.1007/s00453-009-9343-y.

[39] Amitai Perlstein and Rom Pinchasi. Generalized thrackles and geometric graphs in $\mathbb{R}^{3}$ with no pair of strongly avoiding edges. Graphs Combin., 24(4):373-389, 2008.

[40] Rom Pinchasi and Radoš Radoičić. Topological graphs with no selfintersecting cycle of length 4. In Towards a theory of geometric graphs, volume 342 of Contemp. Math., pages 233-243. Amer. Math. Soc., Providence, RI, 2004.

[41] K. S. Sarkaria. A one-dimensional Whitney trick and Kuratowski's graph planarity criterion. Israel J. Math., 73(1):79-89, 1991.

[42] Marcus Schaefer. Removing incident crossings. Unpublished Manuscript, 2010. 
[43] Marcus Schaefer, Eric Sedgwick, and Daniel Štefankovič. Recognizing string graphs in NP. J. Comput. System Sci., 67(2):365-380, 2003. Special issue on STOC2002 (Montreal, QC).

[44] Marcus Schaefer, Eric Sedgwick, and Daniel Štefankovič. Computing Dehn twists and geometric intersection numbers in polynomial time. In Proceedings of the 20th Canadian Conference on Computational Geometry, pages 111-114, 2008.

[45] Shakhar Smorodinsky and Micha Sharir. Selecting points that are heavily covered by pseudo-circles, spheres or rectangles. Combin. Probab. Comput., 13(3):389-411, 2004.

[46] Géza Tóth. A better bound for the pair-crossing number. Presentation at the Conference on Geoemtric Graph Theory, Lausanne, Switzerland, 2010.

[47] Géza Tóth. Note on the pair-crossing number and the odd-crossing number. Discrete Comput. Geom., 39(4):791-799, 2008.

[48] Géza Tóth, 2010. Personal communication.

[49] William T. Tutte. Toward a theory of crossing numbers. J. Combinatorial Theory, 8:45-53, 1970.

[50] Pavel Valtr. On the pair-crossing number. In Combinatorial and Computational Geometry, volume 52 of Math. Sci. Res. Inst. Publ., pages 569-575. Cambridge University Press, Cambridge, 2005.

[51] Hein van der Holst. Algebraic characterizations of outerplanar and planar graphs. European J. Combin., 28(8):2156-2166, 2007.

[52] Hein van der Holst. A polynomial-time algorithm to find a linkless embedding of a graph. J. Combin. Theory Ser. B, 99(2):512-530, 2009.

[53] Hein van der Holst and Rudi Pendavingh. On a graph property generalizing planarity and flatness. Combinatorica, 29(3):337-361, 2009.

[54] E. R. van Kampen. Komplexe in euklidischen Räumen. Abhandlungen aus dem Mathematischen Seminar der Universität Hamburg, 9(1):72-78, December 1933.

[55] Hassler Whitney. The self-intersections of a smooth n-manifold in 2n-space. The Annals of Mathematics, 45(2):220-246, 1944.

[56] Wen Jun Wu. On the planar imbedding of linear graphs. I. J. Systems Sci. Math. Sci., 5(4):290-302, 1985.

[57] Wen Jun Wu. On the planar imbedding of linear graphs (continued). J. Systems Sci. Math. Sci., 6(1):23-35, 1986. 